\title{
Alterations of the Coxiella burnetii Replicative Vacuole Membrane Integrity and Interplay with the Autophagy Pathway
}

\author{
María E. Mansilla Pareja ${ }^{1}$, Antonino Bongiovanni ${ }^{2}$, Frank Lafont ${ }^{2 *}$ and María I. Colombo ${ }^{1 *}$ \\ ${ }^{1}$ Laboratorio de Biología Celular y Molecular-Instituto de Histología y Embriología (IHEM), Universidad Nacional de Cuyo, \\ CONICET, Facultad de Ciencias Médicas, Mendoza, Argentina, ${ }^{2}$ Cellular Microbiology and Physics of Infection \\ Group-Center of Infection and Immunity of Lille, Centre National de la Recherche Scientifique, Institut Pasteur of Lille, \\ UMR8204, Institut National de la Santé Et de la Recherche Médical U1019, Lille Regional Hospital, University Center, \\ Universite Lille, Lille, France
}

OPEN ACCESS

Edited by: Eric Ghigo,

Centre National de la Recherche Scientifique (CNRS), France

Reviewed by: Matteo Bonazzi, Centre National de la Recherche Scientifique (CNRS), France Paras K. Anand, Imperial College London, UK

*Correspondence: Frank Lafont

frank.lafont@cnrs.fr

María I. Colombo

mcolombo@fcm.uncu.edu.ar

Received: 13 January 2017 Accepted: 21 March 2017 Published: 24 April 2017

Citation:

Mansilla Pareja ME, Bongiovanni A, Lafont $F$ and Colombo MI (2017)

Alterations of the Coxiella burnetil Replicative Vacuole Membrane Integrity and Interplay with the

Autophagy Pathway.

Front. Cell. Infect. Microbiol. 7:112. doi: 10.3389/fcimb.2017.00112
Coxiella burnetii, the etiologic agent of $Q$ fever, is a Gram-negative obligate intracellular bacterium. It has been previously described that both the endocytic and autophagic pathways contribute to the Coxiella replicative vacuole (CRV) generation. Galectins are $\beta$-galactoside-binding lectins that accumulate in the cytosol before being secreted via a non-conventional secretory pathway. It has been shown that Galectin-3, -8, -9 monitor bacteria vacuolar rupture and endosomal and lysosomal loss of membrane integrity through binding of host glycans exposed in the cytoplasm after membrane damage. Using microinjection of fluorescence-coupled dextrans, a FRET assay, and galectins distribution, we demonstrate that Coxiella infection actually result in transient phagosomal/CRV membrane damage in a Dot/lcm-dependent manner. We also show the association of different adaptor molecules involved in autophagy and of LC3 to the limiting membrane of the CRV. Moreover, we show that upon autophagy inhibition, the proportion of $C R V$ s labeled with galectins and less acidified increases which is associated with bacteria replication impairment. Based on these observations, we propose that autophagy can facilitate resealing of intracellular damaged membranes.

Keywords: Coxiella burnetii, autophagy, galectins, vacuole membrane, damage detection

\section{INTRODUCTION}

The obligate intracellular bacterium Coxiella burnetii, the etiologic agent of Q-fever (Voth and Heinzen, 2007), is a highly infectious human pathogen responsible for a global zoonotic disease called $\mathrm{Q}$ fever. It is found in a wide range of hosts, including livestock and humans. $C$. burnetii inhabits mainly monocytes/macrophages but it can also infect a wide variety of host cells in vitro (Voth and Heinzen, 2007). It directs the biogenesis of a large membrane-bound compartment called "Coxiella replicative vacuole" (CRV). This compartment has late endosomelysosome characteristics. Coxiella transits the endosomal pathway as revealed by the presence of the Rab5 and EEA1 markers at early times of infection (Romano et al., 2007). Afterwards, the phagosome containing C. burnetii interacts with late endosomes and lysosomes as demonstrated by the recruitment of Rab7 (Berón et al., 2002) and the presence of molecules such as LAMP-1, CD63, and mannose-6-phosphate receptor, (Heinzen et al., 1996; Sauer et al., 2005; Beare et al., 2009). Also, the CRV contains active lysosomal hydrolases and cathepsin D indicating that the 
maturing vacuole fuses with lysosomal compartments (Voth and Heinzen, 2007). In addition, it has been demonstrated the participation of several SNAREs (Vamp3, Vamp7, and Vamp8, Syntaxin 17) in homotypic and heterotypic fusion events in order to consolidate the replicative vacuole (Campoy et al., 2011, 2013; McDonough et al., 2013). It has been additionally shown that the Coxiella housing compartment also interacts with the autophagic pathway (Gutierrez et al., 2005; Romano et al., 2007; Newton et al., 2014; Winchell et al., 2014; Kohler et al., 2016). Likewise, two other proteins involved in autophagy such as Rab24 and Beclin1 (Munafó and Colombo, 2002; Vázquez and Colombo, 2010) are also recruited to the CRV. Furthermore, induction of autophagy favors CRV development (Gutierrez et al., 2005; Romano et al., 2007).

Autophagy is a catabolic pathway that allows the degradation and recycling of intracellular components (organelles and proteins) in double membrane vesicles called autophagosomes (Shibutani and Yoshimori, 2014). It is believed that these structures sequester cytosolic material nonspecifically, but there is also a selective autophagic degradation process of various subcellular structures, including protein aggregates, mitochondria, as well as microbes (Tanida, 2011). A critical role for ubiquitin in this process has been demonstrated since adaptor proteins mediate the binding of autophagosome-associated ubiquitin-like proteins (i.e., LC3/GABARAP proteins) to ubiquitinated cargo, targeting the proteins to the autophagosome (Dupont et al., 2010). One of these ubiquitin binding proteins involved in selective autophagy is p62 (sequestosome 1; SQSTM1) which is an LC3-interacting partner and it is usually degraded by autophagy (Bjørkøy et al., 2005; Komatsu and Ichimura, 2010). Another important autophagy receptor is NDP52 (Nuclear Dot Protein $52 \mathrm{kDa}$ ) that shares with p62 the ability to bind LC3 and ubiquitinated cargo simultaneously (Perrin et al., 2004; Birmingham and Brumell, 2006). However, the specific roles of p62 and NDP52 are not fully understood. Recent reports have shown that reducing NDP52 expression resulted in a decrease in the number of autophagosomes containing Salmonella enterica (Thurston et al., 2012). It is important to mention that NDP52 is capable of directing the formation of an autophagic membrane around ubiquitinated bacteria in the cytosol to allow their clearance by the autophagy machinery (Thurston et al., 2009; Verlhac et al., 2015).

Galectins belong to a family of proteins highly conserved through evolution involved in several biological events. They are able to recognize specific carbohydrates in complex macromolecules located on cell membranes or in the extracellular matrix (ECM) through a carbohydrate recognition domain (CRD), which interacts with the structure (Gal $\beta 1 \rightarrow 4$ GlcNAc). According to the arrangement of their carbohydrate binding domains, galectins are classified into subfamilies: (a) Galectins "proto-type": include galectins 1, 2, 5, 7, and 10. These proteins act as homodimers of two identical CRDs. (b) Galectins "chimera-type": perform their function through a dual interaction. They are contacting carbohydrates through a CRD located at the carboxyl-terminal domain and also they interact with other ligands through their amino-terminal domain rich in proline, glycine and tyrosine. The only member of this family is Galectin-3 (Gal3). (c) Galectins with repetitive sequences ("tandem repeat type"): exhibit two structurally distinct CRD, giving them the capability of interacting with dissimilar carbohydrates. This group includes galectins $4,6,8$, and 9.

Most galectins functions are performed extracellularly. These proteins are involved in processes of immunomodulation, cell adhesion, growth regulation, inflammation, embryogenesis, reproduction, metastasis, proliferation, and "splicing." It has been observed that these carbohydrate binding proteins exert their biological effects through specific recognition-linked sugars in membrane receptors and extracellular glycoproteins (Rabinovich and Toscano, 2009; Vasta, 2013).

The mechanism of synthesis and release of galectins is not yet known. They accumulate in the cytoplasm before being secreted by a leader-peptide-independent pathway. Several studies have presented evidence that Gal3 accumulates in structures in close proximity to phagosomes containing Shigella or Listeria (Dupont et al., 2009; Paz et al., 2010). These bacteria lyse their phagosome, arguing that Gal3 may function as a danger receptor for membrane damages (Dupont and Lafont, 2009). It has been found that inflammasome components are associated with the Shigella-containing vacuole remnant membranes (marked with Gal3) that are driven to the autophagic machinery impairing the inflammatory response (Dupont et al., 2009). It was shown that a set of galectins, especially Galectin 8 (Gal8), recognize injured pathogen-containing vacuoles. Interestingly, at early infection times, Gal8 arbitrates the recruitment of NDP52 but at later times of infection ubiquitin is responsible for the recruitment of NDP52 (Thurston et al., 2009). Thus, this study highlights the recruitment of NDP52, mediated by two different molecules in a sequential fashion, to ensure the proper clearance of cytoplasmic Salmonella.

In the present study, we document, using several approaches, damages of the CRVs. Notably, we have found that several members of the galectin family interact with the Coxiella burnetiicontaining vacuole, at different times of infection. Our results also indicate that adaptor molecules involved in autophagyrelated signaling pathways associate to the limiting membrane of the CRV, likely to control distinct host cell responses upon pathogen infection. In addition, our results demonstrate for the first time that in a population of Coxiella-containing vacuoles the CRV membrane is damaged likely causing the leaking of protons and, as a consequence, a transient modification in its acidic $\mathrm{pH}$. We show that impairing autophagy decrease the number of acidic bacteria-containing vacuoles. We propose that the autophagic pathway favors Coxiella infection by contributing to the repair of the transiently damaged replicative compartment.

\section{RESULTS}

\section{The CRVs Present Heterogeneous Acidic Characteristics}

It has been previously demonstrated that C. burnetii transits the endocytic and autophagic pathways generating an acidic and replicative niche with phagolysosomal characteristics which 
develops in a large CRV at $48 \mathrm{~h}$ of infection (Akporiaye et al., 1983; Maurin et al., 1992; Heinzen et al., 1999; Grieshaber et al., 2002).

We and others have used LysoTracker to label the CRV indicating that these vacuoles have an acidic internal environment. To assess whether the entire population of CRVs posesses the same internal acidic characteristics, infected $\mathrm{CHO}$ cells were incubated with LysoSensor green. This membrane permeant dye increases its fluorescence in acidic environments. Living cells labeled with this compound were analyzed by confocal microscopy. To our surprise, we observed that several CRVs were indeed positive for LysoSensor but most of them displayed less or almost no fluorescence (Figures 1A,B), suggesting that the CRVs did not present homogeneous acidic properties at the time of observation.

Given these striking observations, a different experimental approach was used to assess the acidic $\mathrm{pH}$ inside the CRV. pHrodo is a compound that is almost non-fluorescent at neutral $\mathrm{pH}$, and fluoresces brightly in an acidic environment. Thus, this $\mathrm{pH}$ marker allows detecting acidification or neutralization events by monitoring variations in its fluorescence intensity. Therefore, we first verified that the dextran/pHrodo beads that we were using actually responded to the changes in $\mathrm{pH}$, as expected. As shown in the Figures S1A,B, the beads displayed a clear fluorescence intensity dependency according to the $\mathrm{pH}$ of the media. It is well-known that the Coxiella vacuole is highly fusogenic and readily fuses with other endosomes or phagosomes (e.g., bacteria-containing compartments). Therefore, we allowed the cells to endocytose pHrodo dextran or to phagocytose pHrodo-conjugated heatkilled Staphylococcus aureus (SA/pHrodo). CHO cells were infected with C. burnetii and incubated with pHrodo dextran or SA/pHrodo. The acidification of the CRV was visualized by fluorescence live cell imaging. As depicted in Figures 1C-F, pHrodo fluorescence displays heterogeneity between CRVs, suggesting different populations of these vacuoles depending on the $\mathrm{pH}$. Our results indicate that a large percentage (more than $50 \%$ ) of the vacuoles have a $\mathrm{pH} 7$ or above because their low fluorescence intensity (Figures S1D-E). Interestingly, these differences were not dependent on the vacuole diameter (Figure $\mathrm{S} 1 \mathrm{C}$ ), suggesting that the fluorescence variability was not due to a dilution of the fluorochrome in the large CRV. Therefore, we can infer that there are different populations of CRVs with respect to their $\mathrm{pH}$ and that the possible transient loss of acidity in some of them seems to be independent on the size of the vacuole.

In order to assure that the large majority of the vacuoles has incorporated the pHrodo dye, we did some additional controls to the experiments described above. $\mathrm{CHO}$ cells were incubated with $\mathrm{SA} / \mathrm{pHrodo}$. The $S$. aureus particles were previously incubated with Hoechst to label the bacteria. Cells were analyzed by confocal live imaging (Figure S1D). The percentage of S. aureus positive vacuoles (labeled with Hoechst) as well as vacuoles pHrodo positive or negative was determined. As shown in Figure S1E, approximately $90 \%$ of the C. burnetii vacuoles contained S. aureus particles. However, only $40 \%$ of them were labeled with $\mathrm{pHrodo}$, indicating that the $\mathrm{pH}$ of the rest of the vacuoles was not acidic. We also observed that the pHrodo fluorescence
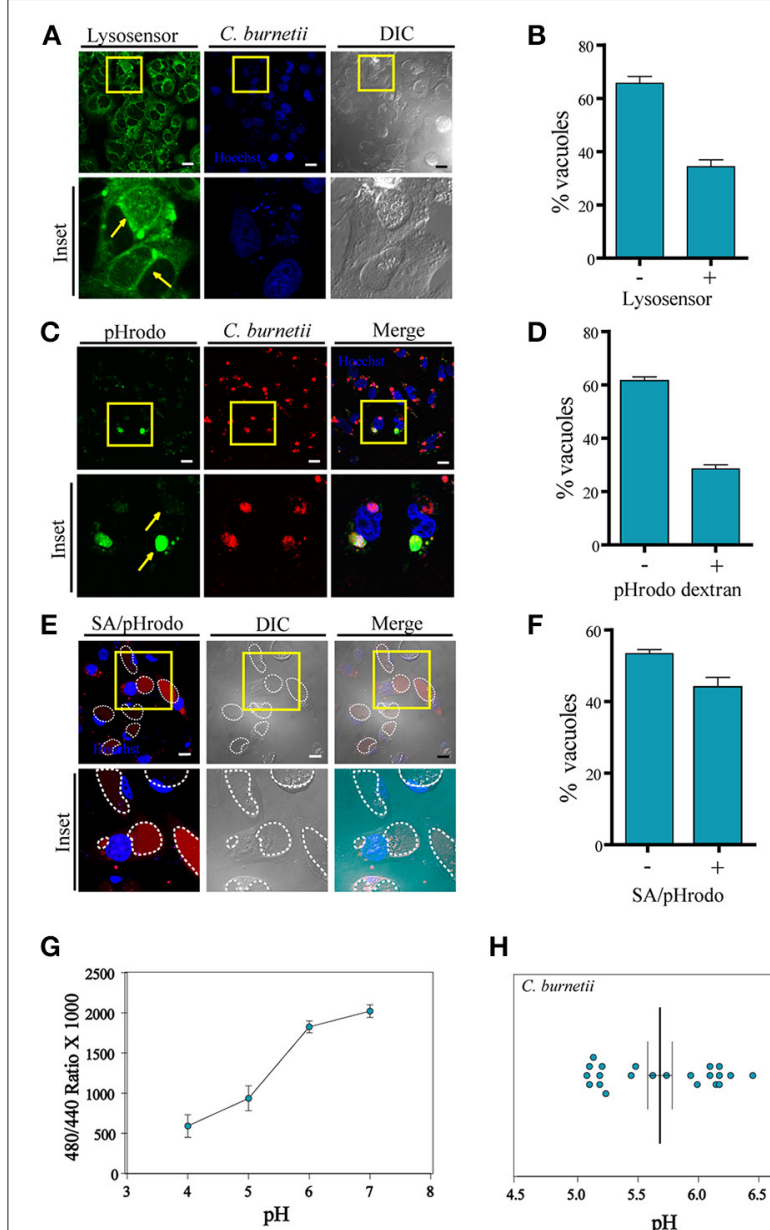

H
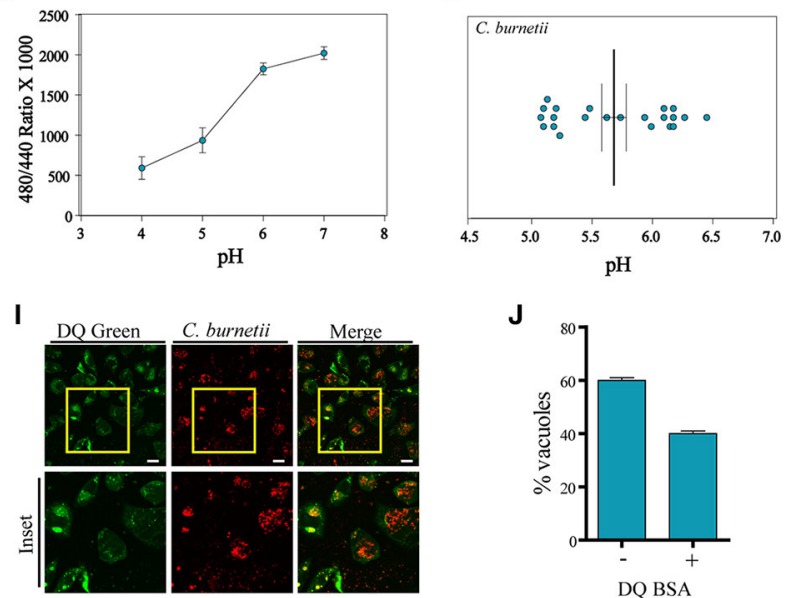

$\mathbf{J}$
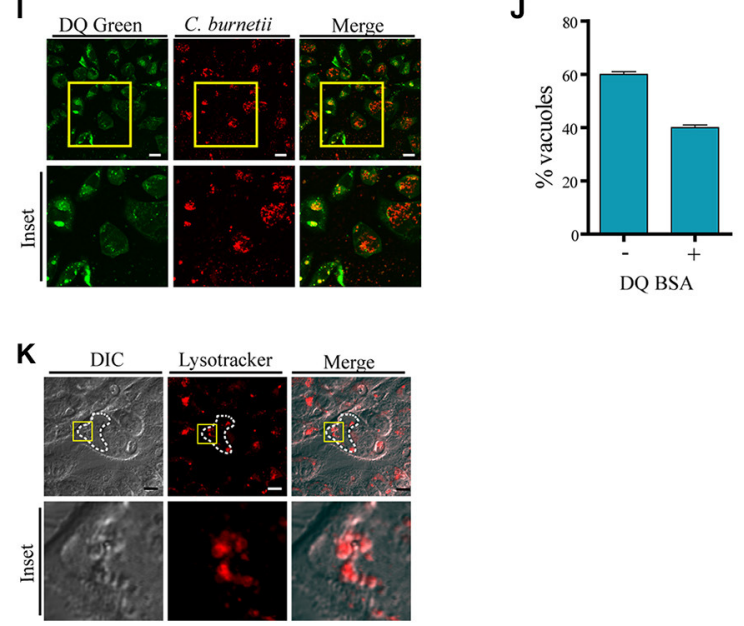

FIGURE 1 | Labeling with pHrodo indicates heterogeneousness of the pH of the CRVs. (A) $\mathrm{CHO}$ cells were infected with C. burnetii. After $48 \mathrm{~h}$, the cells were incubated with Lysosensor green, and analyzed by confocal videomicroscopy. (B) Quantification of the percentage of the vacuoles Lysosensor positive or negative. (C) $\mathrm{CHO}$ cells were infected with $\mathrm{C}$. burnetii. After $48 \mathrm{~h}$, the cells were incubated with pHrodo dextran (green), and analyzed by confocal videomicroscopy. Yellow arrows indicate the differences in fluorescence intensity in two different vacuoles. (D) Quantification of the

(Continued) 


\section{FIGURE 1 | Continued}

percentage of the vacuoles pHrodo dextran positive or negative. (E) $\mathrm{CHO}$ cells were infected with $C$. burnetii. After $48 \mathrm{~h}$, the cells were incubated with pHrodo coupled to dead S. aureus (SA/pHrodo) and analyzed by confocal videomicroscopy. (F) Quantification of the percentage of the vacuoles pHrodo positive or negative. (G) $\mathrm{CHO}$ cells were infected with $\mathrm{C}$. burnetii for $48 \mathrm{~h}$ before loading for $20 \mathrm{~h}$ with Oregon green dextran. The standard curves were generated by measuring the ratio values for the lumenal space of $C$. burnetii's vacuole as described for each of the six $\mathrm{pH}$ standards. These curves were used to determine the $\mathrm{pH}$ within both the Vero cell cytoplasm and the chlamydial inclusion. (H) Experimental ratio values were plotted against the standard curves and of $C$. burnetii replicative vacuoles were calculated. We determined the $\mathrm{pH}$ was $5.68 \pm 0.3(n=22)$ for the $C$. burnetii replicative vacuole. (I) $\mathrm{CHO}$ cells were infected with $\mathrm{C}$. burnetii. After $48 \mathrm{~h}$, cells were incubated with DQ-BSA green and analyzed by confocal videomicroscopy. (J) Quantification of the percentage of the vacuoles DQ green positive or negative. The data represent the mean \pm S.E.M. of at least three independent experiments ( $n>100$ cells/group). (K) $\mathrm{CHO}$ cells were infected with $C$. burnetii. After $48 \mathrm{~h}$, the cells were incubated with LysoTracker red and analyzed by confocal videomicroscopy. Scale bar: $10 \mu \mathrm{m}$.

was dispersed throughout the CCV and not associated with the staph particle. We think this phenomenon is likely due to the release of the dye from the bacterial particles inside the internal environment of the CCV through a mechanism that remains to be determined.

On the other hand, $\mathrm{CHO}$ cells were infected with C. burnetii for $48 \mathrm{~h}$. Then, the cells were co-incubated with Texas red dextran (red), as a control for dextran particles incorporation, and pHrodo dextran (green). Samples were analyzed by confocal microscopy (Figures S1F-G). The percentage of vacuoles Texas red dextran positive (red) or both Texas red dextran and pHrodo dextran positive (red and green) was determined. As indicated in Figure S1G whereas 100\% of the Coxiella vacuoles have incorporated Texas red dextran, only $35-40 \%$ of the vacuoles were also labeled with pHrodo green. This result clearly indicates that the dextran particles were able to reach all the Coxiella vacuoles but only a proportion of those were labeled by pHrodo, indicating that a fraction of the vacuoles were not acidic.

To further verify the $\mathrm{pH}$ of the C. burnetii vacuole, we used ratiometric techniques. The $C$. burnetii vacuole was loaded with Oregon green dextran via fluid-phase endocytosis which has spectral properties that respond to low $\mathrm{pH}$. C. burnetii infected $\mathrm{CHO}$ cells were loaded for $20 \mathrm{~h}$ with Oregon green dextran at a concentration of $1 \mathrm{mg} / \mathrm{ml}$. The $\mathrm{pH}$ for the vacuole was calculated to be $5.68 \pm 0.3(n=22$; Figure $1 G)$. We found that there are two different populations in Coxiella vacuoles $\mathrm{pH}$, as represented in Figure $\mathbf{1 H}$, supporting the existence of intact vacuoles with acidic $\mathrm{pH}$ around 5.2 and transient damaged ones with less acidic $\mathrm{pH}$. This experiment was performed in CHO cells whereas in Akporiaye et al. (1983) and Maurin et al. (1992), macrophages were used. In Akporiaye et al. (1983), the experiment was performed with C. burnetii phase I in J774 cells and authors determined that the $\mathrm{pH}$ was $5.21 \pm 0.07$, whereas in Maurin et al. (1992), the $\mathrm{pH}$ in infected P388DI cells was 4.85 \pm 0.3 . On the other hand, in Vero cells, the $\mathrm{pH}$ of C. burnetii's vacuole was $4.88 \pm 0.14$ as shown in the study by Grieshaber et al.
(2002). Thus, it is possible that the differences observed in the measurement of the $\mathrm{pH}$ in the mentioned papers can be due to the cell type and/or the Coxiella burnetii type used.

To assess the degradative properties of the CRV, CHO cells were infected with C. burnetii for $48 \mathrm{~h}$ and then incubated with DQ-BSA green, a fluorescent marker for degradative compartments. Cells were visualized by fluorescence live cell imaging. As depicted in Figures 1I,J populations of CRVs labeled or not with DQ-BSA green were observed, in agreement with the existence of heterogeneous populations of the Coxiella replicative niche.

In addition, we performed a staining of infected C. burnetii $\mathrm{CHO}$ cells with LysoTracker (red). Consistently with the nonhomogeneous distribution of the LysoTracker labeling, the presence of small vesicles inside the CRV marked by both probes was clearly observed (Figure 1K), confirming the multivesicular nature of the Coxiella-vacuole.

All together these results clearly indicate that at a given time point during infection, the CRVs are highly heterogeneous not only regarding to their acidic $\mathrm{pH}$ but also degradative properties.

\section{The CRV Membrane is Permeable to Cytoplasmic Macromolecular Markers}

We reasoned that the presence of different populations of C. burnetiis vacuole with respect to their $\mathrm{pH}$ indicated that these vacuoles were damaged allowing passive movements of molecules across the CRV membrane. We hypothesized that the observed drop in acidity was a consequence of the leaking of protons through a damaged limiting CRV membrane.

To ascertain whether C. burnetii containing vacuoles were permeable to cytoplasmic markers in a live cell system, soluble dextran of $3 \mathrm{kDa}$ coupled to the Texas Red fluorochrome was microinjected into the cytoplasm of $\mathrm{CHO}$ cells previously infected with $C$. burnetii for $48 \mathrm{~h}$. Vacuolar membrane permeability can be measured through the access of dextrans loaded into the cytoplasm to the CRV. The presence of dextran inside the vacuole was assessed by determining the fluorescence signal inside the CRV, using a spinning disk microscope. For each image of microinjected cells, a sequential optical series ( $\mathrm{z}$ series) and time lapse images were recorded to determine the signal inside the CRV. Tagged dextrans of $3 \mathrm{kDa}$ in size were able to gain access to vacuoles containing live C. burnetii. Figure 2A shows two images of one optical slice of the $\mathrm{z}$ serie and time lapse taken in each microinjection experiment. Figure 2A shows that Texas red dextran entered the CRVs labeled as CRV1 and CRV2. In contrast, the fluorescent marker was excluded from the CRV3 depicted in Figure 2B. The quantification of the fluorescence intensity inside each vacuole is depicted in Figure 2B. The differences among the three selected CRVs are clearly depicted in the insets shown in Figure 2C. The percentage of vacuoles accessed by the microinjected dextran is shown in Figure 2D. Thus, 20\% of the vacuoles presents a relatively substantial damage of the limiting membrane. As a control of microinjection, a $200 \mathrm{kDa}$ dextran was microinjected in the cytoplasm of infected $\mathrm{CHO}$ cells and the cells were visualized by confocal imaging as before. As depicted in the 


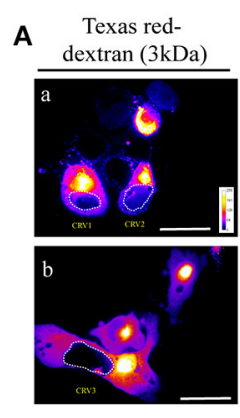

C

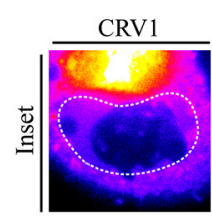

E
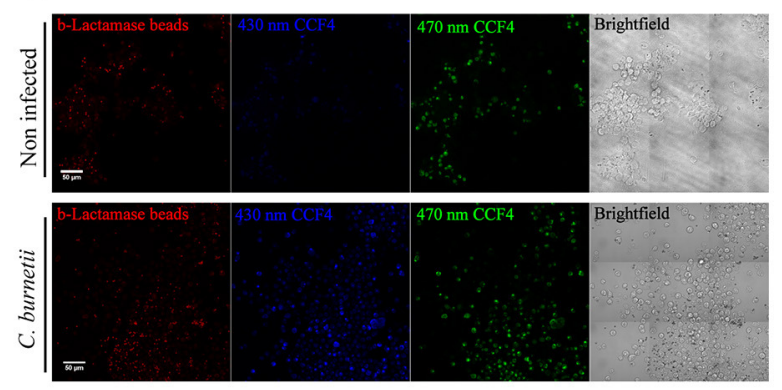

F

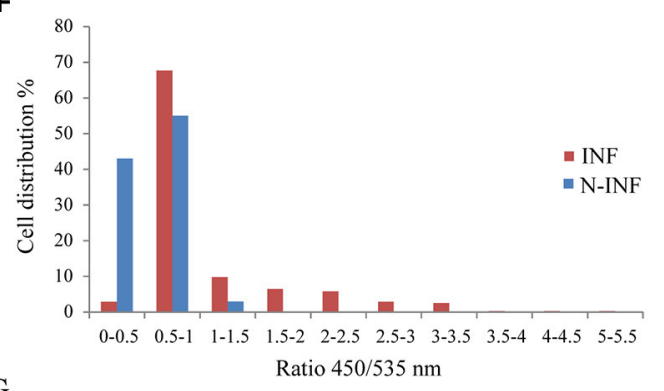

G

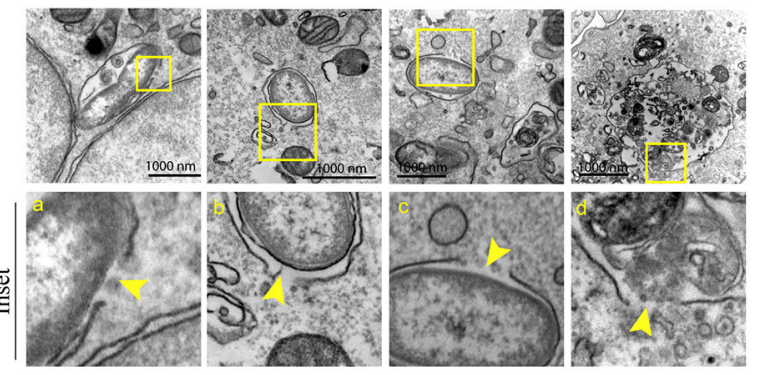

FIGURE 2 | Coxiella damages the endo/phagosomal membranes in infected cells. (A) $\mathrm{CHO}$ cells were infected with $\mathrm{C}$. burnetii for $48 \mathrm{~h}$ and subsequently microinjected with $3 \mathrm{kDa}$ Texas Red-tagged dextrans. Cells were immediately visualized by confocal videomicroscopy. (B) Quantification of the fluorescence intensity of the CRVs indicated in (A). (C) Insets of the vacuoles CRV1, CRV2, and CRV3 shown in (A). (D) Quantification of the percentage of

(Continued)

\section{FIGURE 2 | Continued}

vacuoles dextran positive or negative in microinjected cells. (E) $\mathrm{CHO}$ cells were infected with C. burnetii for $48 \mathrm{~h}$ and loaded with the CCF4 substrate for $2 \mathrm{~h}$. Afterwards, cells were allowed to internalize $1 \mu \mathrm{m}$ latex beads coupled with $\beta$-lactamase and imaged by fluorescence wide field confocal microscope.

Picture acquisition was done randomly and automatically for each condition on 36 fields to follow the FRET signals and the fluorescent beads. Representative pictures were chosen with the following channels: $\beta$-lactamase beads (red), CCF4 cleaved probe $450 \mathrm{~nm}$ (blue) and CCF4 intact probe $535 \mathrm{~nm}$ (green). Scale bar: $50 \mu \mathrm{m}$. (F) Histogram representing the outcome obtained via specialized algorithms using the Image J software. The individual cells are distributed in function of their ratio of the intensities in the 450 and $535 \mathrm{~nm}$ channels. INF: Coxiella infected cells; N-INF: uninfected cells. The plots are representative of 3 independent experiments. (G). CHO cells were infected with $C$. burnetii for $24 \mathrm{~h}$ and then processed for TEM. (a-d) show a bacterium inside a membrane disrupted phagosome. Arrowheads show the point where the membrane is damaged. The insets (a-d) show the higher magnification images of the structures shown in the corresponding top panel.

Figure S2A, this dextran could not get into de CRV, which means that vacuoles were not permeable to this size of molecules and that the microinjection procedure itself was not damaging the CRV.

In order to assure that the vacuoles are permeable, we had to adapt a FRET based reporter assay that was previously used to monitor the intracellular localization of Salmonella and Shigella species (Keller et al., 2013). In the host cell, membrane permeable CCF4-AM molecules diffuse freely across the cellular plasma membrane, gain access to the cytoplasm and are excluded from endosomes and other organelles by anion conversion into CCF4AM upon cytosolic esterase action. The use of this probe relies on the bacterial expression and exposure of beta-lactamase. When the beta-lactamase gets in contact with the cytosolic substrate there is a switch in the FRET signal from $535 \mathrm{~nm}$ (green) to $450 \mathrm{~nm}$ (blue) upon $405 \mathrm{~nm}$ excitation, indicating cleavage of CCF4-AM. The change in the FRET signal indicates rupture of the phagosome/vacuole membrane. Ray et al. (2010). For our assay a modified approach was used. CHO cells were infected with Coxiella for $48 \mathrm{~h}$ and allowed to internalize $1 \mu \mathrm{m}$ latex beads coupled with beta-lactamase. Afterwards, cells were incubated with the CCF4 substrate which is loaded in the cytoplasm. As previously demonstrated for M. marinum (Simeone et al., 2012) and other bacteria (Ray et al., 2010), when the beta-lactamase gets in contact with the cytosolic substrate there is a switch in the FRET signal from $535 \mathrm{~nm}$ (green) to $450 \mathrm{~nm}$ (blue), indicating rupture of the phagosome/vacuole membrane. As shown in the Figure 2E almost all the Coxiella infected cells (INF) turned blue whereas the uninfected (N-INF) cells remained green. The quantification of these results, using an algorithm segmenting individual cells and measuring both fluorescent channels, is shown in Figure 2F. High ratios (above 0.5) indicate the damage of the phagosome membrane as clearly depicted in the panel. As shown in the Figures S2B,C some of the incorporated betalactamase beads (red) can be visualized in a Coxiella-containing compartment (green).

In addition, we have also performed transmission electron microscopy of $\mathrm{CHO}$ cells infected with C. burnetii at early times of infection ( $24 \mathrm{~h}$ ). In Figure 2G, we can observe several 
disruptions in both a Coxiella-containing phagosome membrane as indicated by yellow arrowheads.

Altogether these results noticeably indicate that the CRV surrounding membrane integrity is affected and permeable to cytoplasmic loaded compounds.

\section{Galectins are Recruited to C. burnetii-Containing Compartments at Different Infection Times}

It has been reported that $C$. burnetii transits through the endocytic pathway, which delivers the bacteria to the low $\mathrm{pH}$ environment of a lysosome. It is known that CRV maturation to a phagolysosome takes approximately $2 \mathrm{~h}$. Since galectins are considered useful molecules to detect damage of intracellular membranous compartments, we were interested in determining whether, at some time point during infection, the CRV membrane integrity was altered as suggested by the experiments described above. It has been reported that Gal3 and Gal8 detect membrane damage in bacteria's vacuoles like Shigella and Salmonella (Dupont et al., 2009; Paz et al., 2010; Thurston et al., 2012). Thus, we analyzed the association of these galectins with Coxiella phagosomes at early infection times (i.e., $6 \mathrm{~h}$ ) and later infection times once the large vacuole is generated (i.e., 24, $48 \mathrm{~h}$ ). To this aim, we overexpressed these galectins in $\mathrm{CHO}$ cells and infected cells with C. burnetii. At different times of infection $(6,24$, and $48 \mathrm{~h})$, cells were fixed and analyzed by confocal microscopy as shown in Figure 3A. Figure 3B shows the fluorescence intensity diagram along the yellow line across the CRV depicted in Figure 3A inset. We could observe galectins distributed at the limiting membrane and in the interior of the $\mathrm{CRV}$ as is shown in the Figure S3A, using Structured Illumination Microscopy (SIM) image of a transfected GFP-Gal3 CHO cell infected with C. burnetii. The quantification along the time of infection is depicted in Figure 3C, indicating that YFP-Gal8 and -Gal3 were clearly recruited to a population of the CRVs (30$40 \%)$ at all times of infection analyzed. We also screened a panel of other human galectins for their presence at the C. burnetiicontaining vacuole and we determined whether the interaction of these galectins with the CRV was dependent on the time of infection. For this purpose, $\mathrm{CHO}$ cells were transfected for $24 \mathrm{~h}$ with YFP-Gal4, YFP-Gal10, YFP-Gall and YFP-Gal9 and cells were infected for different periods of time. Figure S3C shows confocal images of the kinetic analysis for the Gall, Gal4, Gal9, and Gal10. The population of C. burnetii phagosomes that recruited the tested galectins during the course of infection was also quantified (Figure S3D). As depicted, the colocalization between C. burnetii and YFP-Gal1 increased at $24 \mathrm{~h}$ of infection and it remained constant at $48 \mathrm{~h}$. In contrast, in the case of YFPGal9 and -Gal4 the proportion decreased at later infection times whereas no colocalization along infection was observed in the case of YFP-Gal10.

Since overexpressed Gal3, Gal8, and Gall interact with C. burnetii at different stages of vacuole development and persist along the infection process $(48 \mathrm{~h})$, we were interested in determining whether the endogenous proteins were also recruited at the CRV. Cells were infected with C. burnetii for
$48 \mathrm{~h}$ and both bacteria and endogenous galectins were detected using specific antibodies. As shown in Figure 3D, we observed the accumulation of endogenous Gal3, Gal1, and Gal 8 at the CRV surrounding membrane (insets). The quantification of the percentage of CRVs positive for endogenous Gal3, Gal1, or Gal8 is depicted in Figure 3F. Our results clearly indicate that 25-30\% of the CRVs are decorated by these galectins. Also, there is a large amount of colocalization between Gal3 and Gal8 at early times of infection (6h; Figure 4A). CHO cells were transfected with mCherry-Gal8 and infected with C. burnetii. Then, the cells were fixed and subjected to immunofluorescence with specific antibodies against Gal3. The quantification of colocalization is depicted in Figure 4B, indicating that both galectins are recruited to the same CRV.

In addition, we were interested in determining the presence of galectins at earlier times of infection. For this purpose, CHO cells transfected with YFP-Gal8 or YFP-Gal3 were infected for $2 \mathrm{~h}$ with mCherry-C. burnetii. The cells were analyzed by fluorescence confocal videomicroscopy and a multidimensional image was constructed from a $\mathrm{z}$-interval (Figure 4C). The image clearly shows the recruitment of YFP-Gal8 to a small phagosome containing just a bacterium, confirming that Gal8 associates to the Coxiella phagosome at very early times after infection. Figure 4D, shows the quantification of the recruitment of these galectins at $2 \mathrm{~h}$ of infection.

In order to dynamically image the recruitment of galectins to the Coxiella phagosome, $\mathrm{CHO}$ cells were transiently transfected with GFP-Gal3 and infected with C. burnetii incubated with Hoechst. The cells were analyzed by fluorescence confocal videomicroscopy. Figure $4 \mathrm{E}$ shows an infected cell with an early recruitment of Gal3 around a group of bacteria. The sequence shows images taken every $5 \mathrm{~min}$ in a sequence of the total time lapse analyzed from 0 to $50 \mathrm{~min}$. Hence, there is a recruitment of Gal3 around bacteria starting at the time point $25 \mathrm{~min}$ of infection and remaining during the whole period of time analyzed while the phagosomes become larger.

Moreover, in order to assess whether some galectin-positive compartments have lost their acidity, $\mathrm{CHO}$ cells were transiently transfected with RFP-Gal8. Then, the cells were infected with C. burnetii for $24 \mathrm{~h}$. Afterwards, the cells were incubated with pHrodo green dextran. The acidification of the CRV was visualized by fluorescence live cell imaging (Figure 4F). As depicted in the Figure 4G, the large majority of the mCherry Gal8-positive vacuoles are pHrodo negative. Nevertheless, Coxiella-containing vacuoles positive for Gal8 and pHrodo are also clearly observed. This can be interpreted as a transient membrane damage, hence neutralizing the vacuolar $\mathrm{pH}$, before vacuoles recover acidification as membrane is repaired. In other words, at some point the vacuole was pierced and this damage was detected by galectins but since the damage is transient they recover the acidity but the CRV still remain positive for galectin. Thus, healing the membrane damage may contribute to the recovering of acidity necessary for the Coxiella metabolic activity.

In order to determine whether the galectin recruitment is specifically dependent on bacterial infection, a control experiment with an inert particle was performed. $\mathrm{CHO}$ cells were 

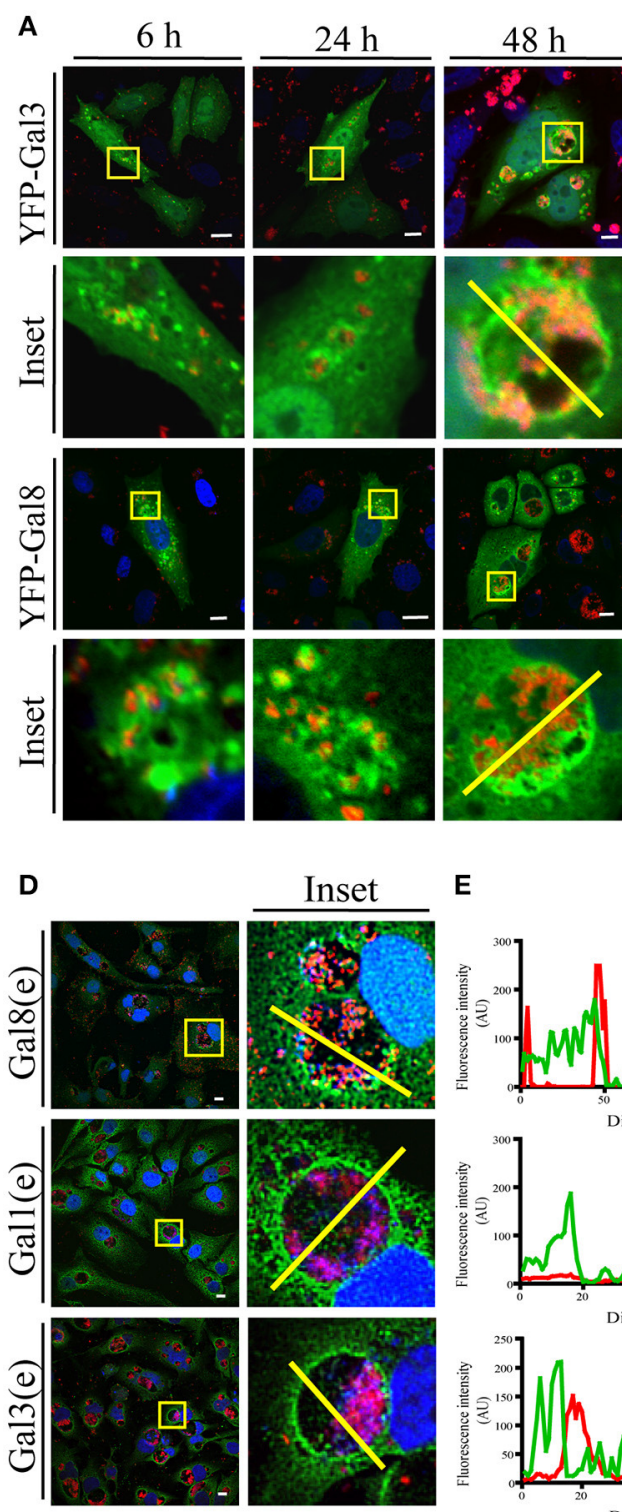

B
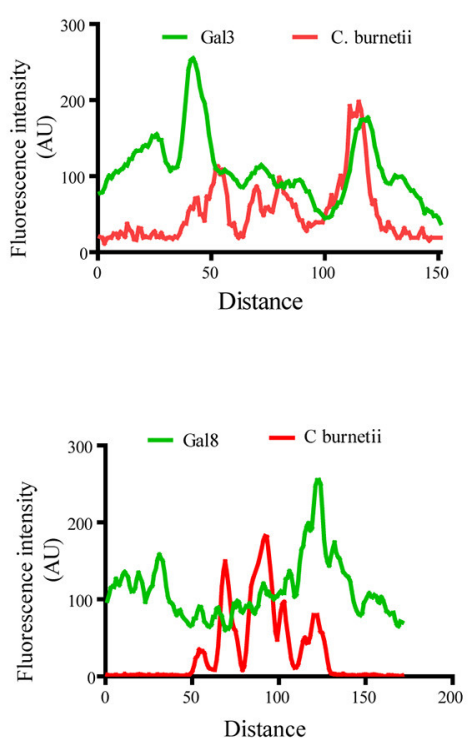

C
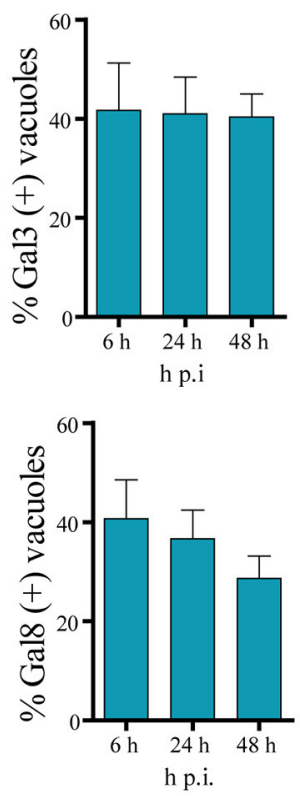

$\mathbf{F}$
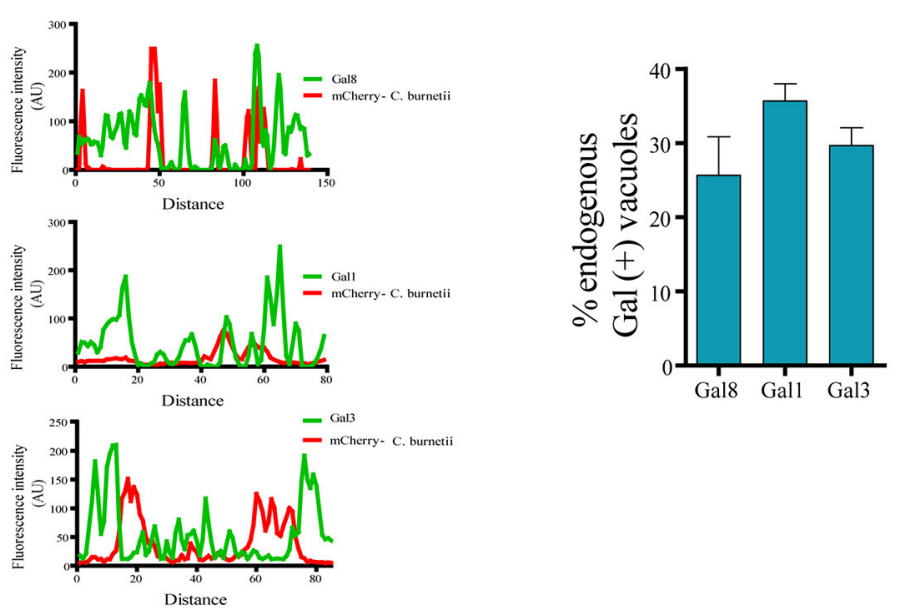

FIGURE 3 | Galectins decorate the CRV membrane. (A) CHO cells were transiently transfected with YFP-Gal3 and -Gal8 and then infected with mCherry-C. burnetii. At 6, 2,4, and 48 h of infection, cells were fixed and analyzed by confocal microscopy. (B) Fluorescent intensity profiles along the yellow line depicted in the corresponding inset of (A) (YFP-Gal3 and -Gal8). (C) Quantification of the percentage of the CRVs labeled with YFP-Gal3 and -Gal8 from images like the ones depicted in (A). The data represent the mean \pm S.E.M. of at least three independent experiments ( $n>50$ cells/group). (D) CHO cells were infected with C. burnetii for $48 \mathrm{~h}$, fixed and subjected to indirect immunofluorescence using antibodies against Gal1, Gal3, and Gal8 to detect the endogenous proteins (green), and an anti-Coxiella to detect the bacterium (red). Cells were analyzed by confocal microscopy. (E) Fluorescent intensity profiles along the yellow line depicted in the corresponding inset of (D). (F) Quantification of the percentage of CRVs labeled with each of the galectins in images like the ones depicted in (D). The data represent the mean \pm S.E.M. of at least three independent experiments ( $n>50$ cells/group). Scale bar: $10 \mu \mathrm{m}$.

transfected with GFP-Gal3 and incubated with rhodamine beads for different times $(2,6,24,48 \mathrm{~h})$, fixed and analyzed by confocal microscopy (Figure S4A). In addition, in a parallel experiment, endogenous Gal3 was determined in $\mathrm{CHO}$ cells incubated with FITC beads at the same time points (Figure S4C). As depicted in the graphs of fluorescence intensity along the yellow line across beads phagosomes, no recruitment of galectins to the beads-containing phagosomes was observed. Figures S4B,D show the quantification of the association of Gal3 to beads phagosomes.

\section{The Recruitment of Galectins is Dependent on Host Cell Glycans}

The damage of the vacuole exposes host glycans which may cause Gal8 and Gal3 accumulation at the CRV. As indicated in the introduction, Gal3 has only one carbohydrate recognition 


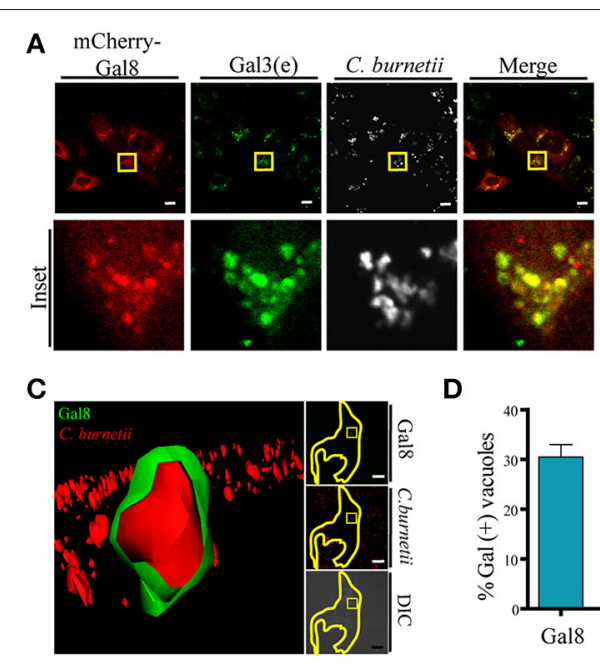

B

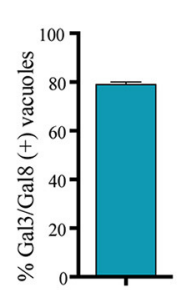

D

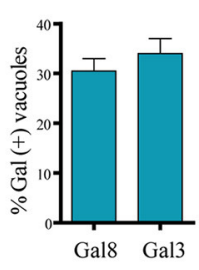

E
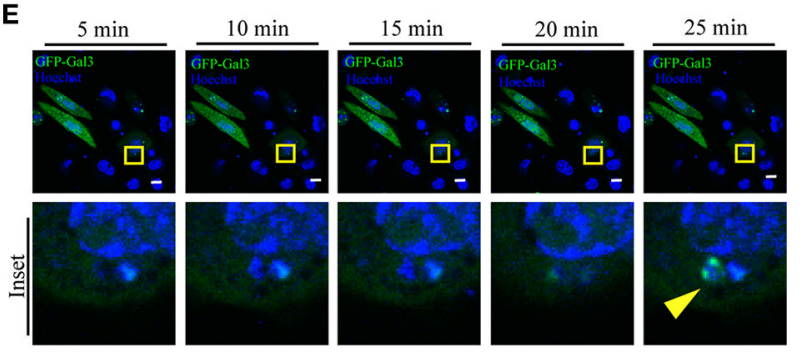

$30 \mathrm{~min}$
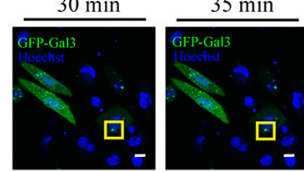

$40 \mathrm{~min}$
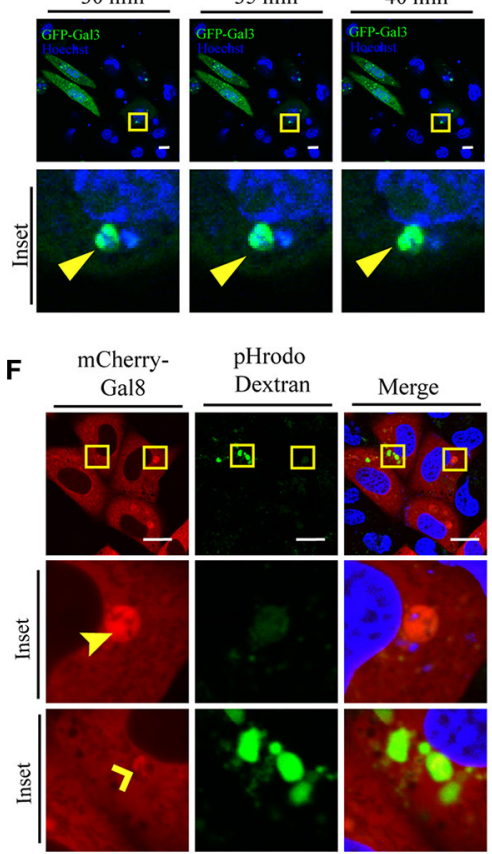

G

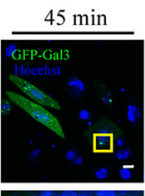

$50 \mathrm{~min}$
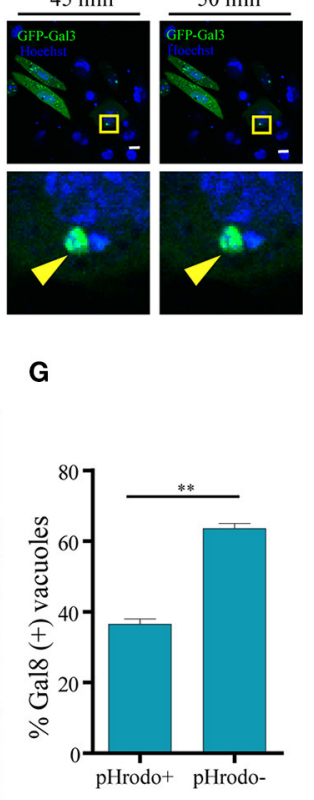

FIGURE 4 | Galectins are recruited to the Coxiella phagosomes at early times of infection. (A) $\mathrm{CHO}$ cells were transiently transfected with mCherry-Gal8 and then infected with C. burnetii (white). At $6 \mathrm{~h}$ of infection, cells were fixed, and subjected to immunofluorescence against Gal3 [endogeneous (e), green]. The cells were analyzed by confocal microscopy. (B) Quantification of percentage of Gal3 positive vacuoles that colocalize with Gal8 in images like the ones depicted in (A). The data represent the mean \pm

(Continued)

\section{FIGURE 4 | Continued}

S.E.M. of at least three independent experiments ( $n>50$ cells/group). (C) $\mathrm{CHO}$ cells were transiently transfected with YFP-Gal8 and then infected with $m$ Cherry-C. burnetii. At $2 \mathrm{~h}$ of infection, cells were and analyzed by confocal videomicroscopy. The inset shows a multidimensional image constructed from a z-interval. (D) Quantification of the percentage of CRVs labeled galectins at $2 \mathrm{~h}$ of infection. The data represent the mean \pm S.E.M. of at least three independent experiments ( $n>50$ cells/group). (E) $\mathrm{CHO}$ cells transiently overexpressing GFP-Gal3 were infected with $C$. burnetii $(\mathrm{MOI}=50)$. The cells were analyzed by fluorescence microscopy for $2 \mathrm{~h}$ in vivo. Insets of the experiment show the sequences of the movie (every $5 \mathrm{~min}$ up to $50 \mathrm{~min}$ ). Yellow arrows indicate the small $C$. burnetii-containing phagosome positive for GFP-Gal3. (F) CHO cells were transfected with mCherry-Gal8 and at $24 \mathrm{~h}$ post transfection were infected for $24 \mathrm{~h}$ with C. burnetii. Coxiella-infected cells were incubated with pHrodo dextran (green) for $3 \mathrm{~h}$ and visualized by confocal microscopy. (G) Quantification of the percentage of Gal8-positive vacuoles that were either $\mathrm{pH}$ rodo dextran positive $(+)$ or negative $(-)$. The data represent the mean \pm S.E.M. of at least three independent experiments $(n>$ 50 cells/group) $\left({ }^{* *} P \leq 0.01\right)$. Scale bar: $10 \mu \mathrm{m}$.

domain (CRD) whereas Gal8 belongs to the group of "tandem repeat type" galectins that exhibit two structurally distinct CRDs, being able to interact with dissimilar carbohydrates. The requirement for carbohydrate binding by Gal8 and Gal3 was tested using point mutations in their respective CRDs. $\mathrm{CHO}$ cells were transfected with the corresponding carbohydratebinding mutants YFP-Gal8 (R275H), YFP-Gal8 (R69H) and GFP-Gal3 (R186S). All these mutants carry a mutation in one critical aminoacid at the CRD so that they are not able to recognize carbohydrates. Afterwards, the cells were infected with $C$. burnetii and, at $48 \mathrm{~h}$ of infection they were fixed and analyzed by confocal microscopy (Figures $\mathbf{5 A}, \mathbf{B}$ ). As shown in the quantification depicted in Figure 5C, in contrast to the wt YFP-Gal8 or GFP-Gal3, none of the mutants did accumulate at the CRD, indicating that either the Gal3 CRD or both Gal8 CRD domains are absolutely required for recruitment to the Coxiella vacuoles.

Furthermore, to demonstrate that host glycans are indeed important for the recruitment of galectins to the CRV, we transfected $\mathrm{CHO}$ and $\mathrm{CHO}-\mathrm{Lec} 3.2 .8 .1$ cells, which lack mature glycans, with mCherry-Gal8. Transfected cells were infected with GFP-C. burnetii and at $48 \mathrm{~h}$ of infection, cells were fixed and analyzed by confocal microscopy (Figure 5D). As shown in Figure 5E, recruitment of Gal8 to CRVs was severely impaired in the CHO-Lec3.2.8.1 cells. This indicates that the presence of mature glycans of the host cell is important for the recruitment of Gal8 to the CRV.

To assess whether Gal3 inside C. burnetii-containing vacuole may arrive from the plasma membrane when C. burnetii is phagocytosed, we utilized the reagent N-Acetyl-D-lactosamine, which blocks the interactions between Gal3 and its ligands at the cell surface. $\mathrm{CHO}$ cells were incubated with (N-AcetylD-lac) or without (Control) the inhibitor and after $2 \mathrm{~h}$, the cells were infected with C. burnetii (red) for $48 \mathrm{~h}$ in the continuous presence of the inhibitor. Afterwards, the cells were fixed and endogenous Gal3 (green) was detected by immunofluorescence and analyzed by confocal microscopy (Figures 5F,G). The same experiment was performed with 

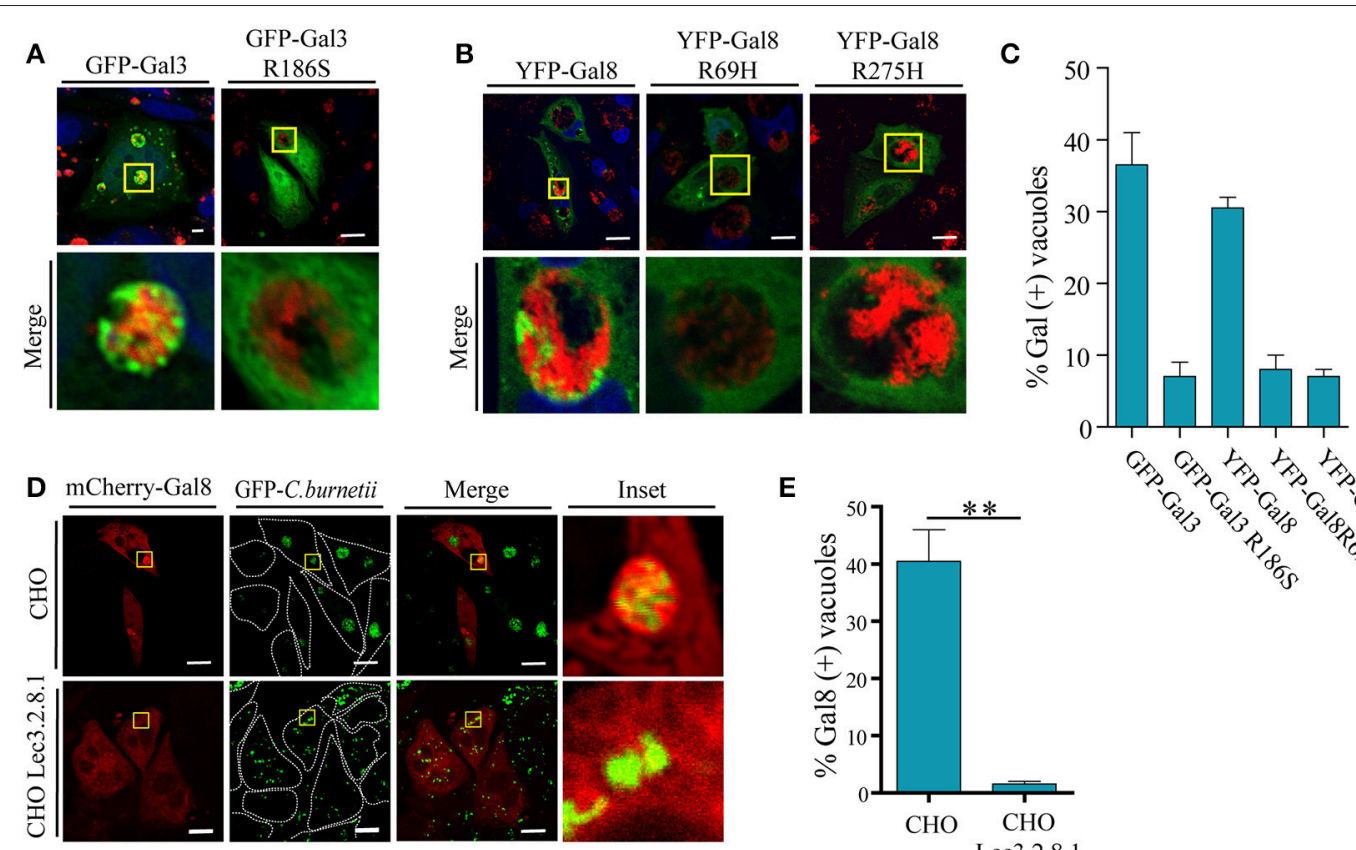

E
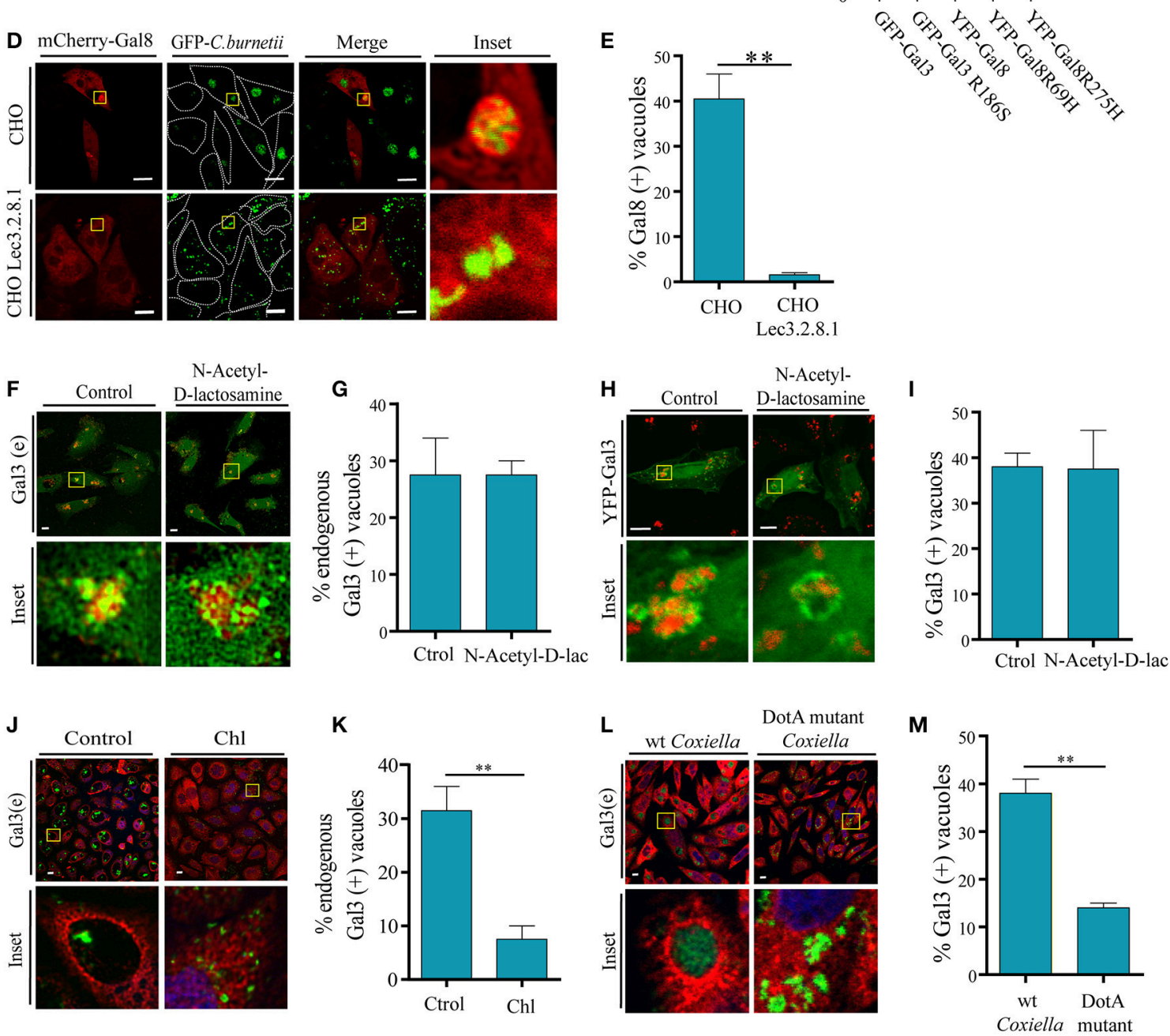

FIGURE 5 | Galectins recruitment is dependent on the binding to host glycans and on a bacterial secretion system. (A) CHO cells were transiently transfected with YFP-Gal3 and YFP-Gal3 G186S and then infected with mCherry-C. burnetii. At $48 \mathrm{~h}$ of infection, cells were fixed and analyzed by confocal microscopy. (B) CHO cells were transiently transfected with YFP-Gal3, YFP-Gal8 R69H, and YFP-Gal8 R275H and then infected with $m C h e r r y-C$. burnetii. At 48 h of infection, cells were fixed and analyzed by confocal microscopy. (C) Quantification of the percentage of CRVs labeled with each of the galectins in images like the ones depicted in (A,B). The data represent the mean \pm S.E.M. of at least three independent experiments ( $n>50$ cells/group). (D) CHO and CHO Lec3.2.8.1 cells were transiently transfected with mCherry-Gal8 and then infected with GFP-C. burnetii. At $48 \mathrm{~h}$ of infection, cells were fixed and analyzed by confocal microscopy. (E) Quantification of the percentage of CRVs labeled Gal8 from images depicted in (D). (F) CHO cells were incubated with N-Acetyl-D-lactosamine. After $2 \mathrm{~h}$, cells were infected with $C$. burnetii (red) maintaining the reagent in the culture medium and, at $48 \mathrm{~h}$, the cells were fixed and subjected to immunofluorescence using specific antibodies against Gal3 (green) and analyzed by confocal microscopy. (G) Quantification of the percentage of CRVs labeled Gal3 in each condition (Control and incubated with N-Acetyl-D-lactosamine) from images like the ones depicted in (F). (H) CHO cells were transiently transfected with YFP-Gal3 and then they were incubated with N-Acetyl-D-lactosamine. After $2 \mathrm{~h}$, cells were infected and at $48 \mathrm{~h}$, the cells were fixed and analyzed by confocal microscopy. (I) Quantification of the percentage of CRVs labeled Gal3 in each condition (control and incubated with N-Acetyl-D-lactosamine) from images like the ones depicted in (H). (J) CHO cells were 
FIGURE 5 | Continued

infected with either untreated (control) or treated Coxiella with $100 \mu \mathrm{g} / \mathrm{ml}$ of chloramphenicol (Chl) for $30 \mathrm{~min}$, cells were incubated for $24 \mathrm{~h}$, fixed and subjected to indirect immunofluorescence using specific antibodies against C. burnetii (green) and Gal3 (red).Cells were analyzed by confocal microscopy. (K) Quantification of the percentage of CRVs labeled with Gal3 from images like the ones depicted in (J). (L) CHO cells were infected with GFP-C. burnetii and GFP-C. burnetii DotA mutant. At $48 \mathrm{~h}$ of infection, the cells were fixed and subjected to immunofluorescence using specific antibodies against Gal3 (red). The cells were analyzed by confocal microscopy. (M) Quantification of the percentage of CRVs labeled Gal3 from images like the ones depicted in (G). The data represent the mean \pm S.E.M. of at least two independent experiments $\left(n>50\right.$ cells/group) $\left.{ }^{\star \star} P \leq 0.01\right)$. Scale bar: $10 \mu \mathrm{m}$.

YFP-Gal3 transfected $\mathrm{CHO}$ cells (Figures 5H,I). We could observe the presence of Gal3 recruited to the vacuole in both control conditions and when incubated with the inhibitor $(\mathrm{N}-$ Acetyl-D-lac). This result indicates that the Gal3 present at the CRV does not come from the plasma membrane but from the cytoplasm.

\section{The Recruitment of Galectins Is Dependent on C. burnetii Secretion System}

It has been shown that $C$. burnetii possesses a type 4 secretion system (T4SS) termed Dot and Icm. Recent studies have demonstrated that $\mathrm{Dot} / \mathrm{Icm}$ function is required for cytosolic delivery of several substrates which have been identified as proteins effectors important for intracellular replication, development of the large CRV and apoptosis protection of infected THP-1 cells. Nevertheless, it was found that translocation of Dot/Icm substrates is not required for axenic growth or uptake by host cells (Carey et al., 2011; Newton and Roy, 2011; Beare et al., 2012).

In previous reports, it has been demonstrated that T4SS function can be inhibited by chloramphenicol treatment. In order to assess whether the association between C. burnetii and galectins is an active phenomenon that depends on bacterial protein synthesis, C. burnetii was pretreated with $100 \mu \mathrm{g} / \mathrm{ml}$ of chloramphenicol for $1 \mathrm{~h}$ and $\mathrm{CHO}$ cells were then infected for $48 \mathrm{~h}$, with the continuous presence of the antibiotic in the culture medium. Cells were fixed and subjected to a double immunofluorescence, using specific antibodies against $C$. burnetii and Gal3 (Figure 5J). Consistently with the results presented above, we observed in the control condition that a colocalization of about $30-40 \%$ of the CRV recruited this galectin, whereas with the chloramphenicol-treated bacteria the percentage of colocalization was significant smaller (around 10\%; Figure 5K). This result clearly indicates that the recruitment of galectins depends on an active protein synthesis by C. burnetii. To actually demonstrate that galectin recruitment is dependent on T4SS, CHO cells were infected with GFP-wt C. burnetii or a DotA mutant GFP- C. burnetii (Tn292). This mutant carries an independent transposon insertion in the gene CBU_1648, which encodes DotA, an essential component of the Coxiella Dot/Icm secretion system. At $48 \mathrm{~h}$ of infection, cells were fixed and subjected to immunofluorescence against endogenous Gal3 (red). Similar to chloramphenicol treatment, cells infected with DotA mutant did not recruit Gal3 compared to control wt Coxiella (Figures 5L,M). These results show that C. burnetii's $\mathrm{Dot} / \mathrm{Icm}$ secretion system is important for the recruitment of galectins.

\section{NDP52 Is Present at the CRV and Colocalizes with Both LC3 and Gal3 or Gal8}

It has been shown in previous publications that Salmonella enterica serotype Typhimurium and Streptococcus pyogenes are recognized by NDP52 and recruits polyubiquitinated proteins once in the cytosol (Thurston et al., 2009). NDP52 bridges LC3 and ubiquitin molecules around bacteria allowing the transport of cytosolic bacteria to the autophagic pathway. Based on these evidences, we next assessed the association of overexpressed RFPNDP52 at the CRV. As shown in Figures S5A,B, the results indicate that the protein was found in $20-30 \%$ of the vacuoles generated after $48 \mathrm{~h}$. In order to determine whether galectins also colocalized with NDP52 in the same Coxiella vacuole, YFPGal8 or YFP-Gal3 and RFP-NDP52 were coexpressed in CHO cells which were subsequently infected with C. burnetii for $48 \mathrm{~h}$. As shown in Figures S5C,D, we found that around $80 \%$ of the galectin-positive CRVs accumulated NDP52. Furthermore, NDP52 also markedly colocalized with LC3 in the large vacuoles generated at $48 \mathrm{~h}$ infection (Figures S5E,F). The results suggest that galectin-dependent recruitment of NDP52 to the CRV is likely to participate to the further association of LC3.

The protein p62 (also known as SQSTM1) targets intracellular pathogens for degradation in autophagolysosomes. P62 is a receptor for cargo with multiple protein-protein interaction domains that binds ubiquitin and also LC3 (Komatsu and Ichimura, 2010). Because NDP52 was recruited to the C. burnetii's CRV, we also evaluated the presence of p62 at the vacuole membrane. $\mathrm{CHO}$ cells were infected with mCherry-C. burnetii for $48 \mathrm{~h}$ and the cells were subjected to immunofluorescence with a specific antibody against p62. In the Figure S6A the association of p62 to the CRV membrane is depicted. Figure S6B shows the quantification of the percentage of vacuoles that recruit p62. Thus, we were next interested to also determine the presence of ubiquitin to the vacuoles. CHO cells were infected with C. burnetii for $48 \mathrm{~h}$ and then the cells were fixed. As depicted in Figure S6C, the vacuoles were decorated by ubiquitin (see quantification in Figure S6D) suggesting that $C$. burnetii recruits in an ubiquitin-dependent manner adaptors of the autophagy machinery.

\section{The Population of CRV Galectin Positive Vacuoles Increases in Cells with a Defective Autophagy Pathway}

In several previous publications it has been shown the association of LC3 to the large CRV (Berón et al., 2002; Gutierrez et al., 2005; Romano et al., 2007). Therefore, we next examined whether both Gal3 or Gal8 and LC3 were recruited to the same vacuole. To 
examine in details the respective distribution of galectins and LC3 during C. burnetii infection, cells were co-transfected with YFP-Gal3 or YFP-Gal8 and RFP-LC3 and they were infected with C. burnetii for $48 \mathrm{~h}$. Figure S5G clearly shows the presence of either YFP-Gal3 or YFP-Gal8 and RFP-LC3 decorating the large CRV. The image shows the accumulation of LC3 (red) and galectins (green) at the surrounding CRV membrane but also in small structures inside the CRV that may represent small internal vesicles (Figure S5G insets). Figure S5H shows the quantification of LC3 positive vacuoles (from a total around of 30\%) that colocalize with Gal8 or Gal3. We can conclude that in $48 \mathrm{~h}$ vacuoles, galectins and LC3 are both present suggesting that similar to other pathogens galectins may mediate the recruitment of LC3 to the CRV.

In order to assess whether the recruitment of Gal3 was modulated by the autophagic pathway, $\mathrm{CHO}$ cells overexpressing Gal3 were infected with C. burnetii duting $48 \mathrm{~h}$. Then, the cells were subsequently incubated in full nutrient media (Control) or under starvation conditions (Stv) or with Rapamycin (Rap) to induce autophagy. As shown in Figure S5J, no major differences were observed in the recruitment of Gal3 in all the conditions tested.

To directly assess the role of autophagy in galectins recruitment, we used a mouse embryonic fibroblast (MEF) cell line harboring a knock-out for the atg5 locus (atg5 -/-). These cells lacking Atg5 are deficient in macroautophagy because this protein is essential for the early steps of autophagosome formation. To demonstrate the significance of the membrane damaged caused by Coxiella and the role of autophagy in this process, MEF wt and Atg5-/- cells were infected with C. burnetii and following $48 \mathrm{~h}$ of infection, the cells were fixed, subjected to immunofluorescence against Gal3 (red) and analyzed by confocal microscopy. As expected, in control cells, endogenous Gal3 was recruited to a population of the CRVs. However, in MEF Atg5-/- cells, a higher percentage of vacuoles decorated by endogenous Gal3 were observed, indicating that a larger number of CRVs were damaged and that autophagy is likely required for membrane repair (Figures 6A,B). Similar results were also observed when cells MEF wt or Atg5-/ - overexpressing YFP-Gal3 were infected with C. burnetii confirming that this galectin was recruited to the vacuole in an autophagy-independent manner (Figures 6C,D). However, the number of CRV positive for galectin was dependent on a functional autophagy pathway.

As indicated above pHrodo is a fluorogenic dye that becomes highly fluorescent as the $\mathrm{pH}$ decreases, therefore, it is a useful probe to assess acidic compartments. To corroborate that in autophagy incompetent cells more vacuoles were actually damaged and, as a consequence, the acidity of the CRV was lost due to the leaking of protons, we used pHrodo as a marker of acidic vacuoles. For this purpose, MEF wt and Atg5-/infected cells were incubated with pHrodo-conjugated to heatkilled Staphylococcus aureus to be internalized and visualized by fluorescence live cell imaging. As depicted in Figure 6E, in Atg5-I- cells, there was a decrease in the number of vacuoles labeled with the fluorogenic compound, suggesting that there were less acidic vacuoles in autophagy-deficient cells. This result

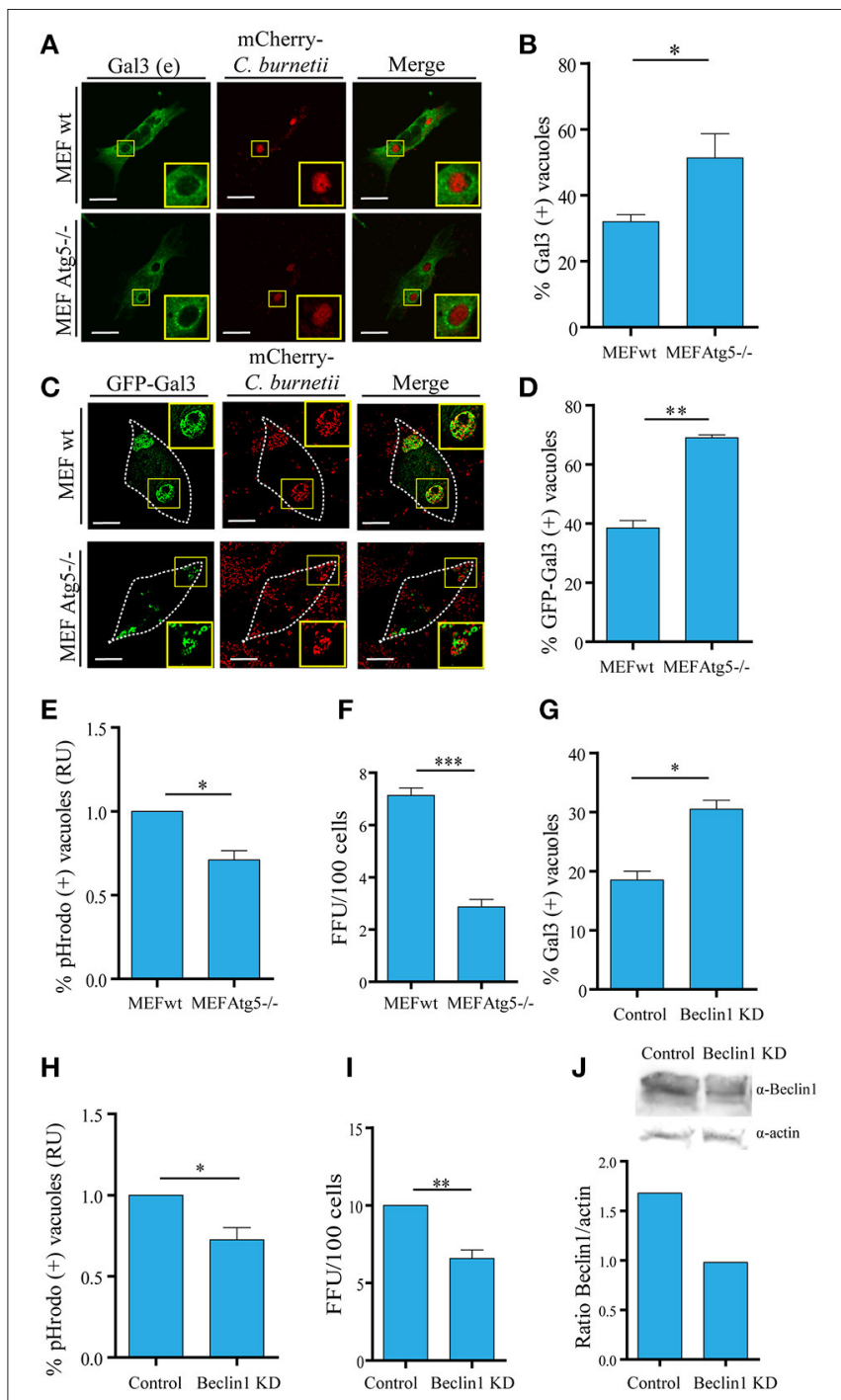

FIGURE 6 | Autophagy contributes to keep the integrity of the vacuolar membrane. (A) WT and Atg5 -/- MEF cells were infected with $C$. burnetii (red) and, at $48 \mathrm{~h}$ of infection, cells were subjected to indirect immunofluorescence with specific antibodies against Gal3. (B) Quantification of Gal3-positive vacuoles in MEF wt and Atg5-/- cells is shown. (C) WT and Atg 5-/- MEF cells were transfected with GFP-Gal3. After $24 \mathrm{~h}$ post-transfection, cells were infected with $C$. burnetii (red) for $48 \mathrm{~h}$. Cells were fixed, and examined by confocal microscopy. (D). Quantification of Gal3-positive vacuoles in MEF wt and Atg5-/- cells is shown. Scale bar: 10 $\mu \mathrm{m}$. (E) Quantification of the pHrodo-positive vacuoles in MEF wt and Atg5-/- cells infected with C. burnetii. After $48 \mathrm{~h}$, cells were incubated with pHrodo, and analyzed by confocal videomicroscopy. Data represent the mean \pm SEM of at least three independent experiments in which no $<200$ vacuoles were scored in each experiment $\left({ }^{*} P \leq 0.05\right)$. (F) MEF wt and MEF Atg5-/cells were infected with $C$. burnetii and following $48 \mathrm{~h}$ of infection to allow the development of the Coxiella vacuole. The cells were then lysed by sonication and the supernatant was diluted $(1: 100)$ and used to infect Vero cells. After $72 \mathrm{~h}$ of incubation (chase), cells were fixed and examined by fluorescence microscopy. Data represent the mean \pm SEM of at least three independent experiments where at least 1,000 cells were scored in each experiment ${ }^{* \star *} P \leq$ 0.001). (G) HeLa cells were transfected with Beclin $1 \mathrm{KD}$ plasmid using a double-hit protocol. Control and Beclin $1 \mathrm{KD}$ cells were

infected with C. burnetii and, at $48 \mathrm{~h}$ of infection, cells were subjected to indirect

(Continued) 


\section{FIGURE 6 | Continued}

immunofluorescence with specific antibodies against Gal3. Cells were fixed, and examined by confocal microscopy. Quantification of Gal3-positive vacuoles in control and Beclin1 KD cells is shown. $(\mathbf{H})$ Quantification of the pHrodo-positive vacuoles in HeLa control and Beclin1 KD cells infected with C. burnetii. Cells were treated and infected as described in (G). After $48 \mathrm{~h}$, cells were incubated with pHrodo and analyzed by confocal videomicroscopy. Data represent the mean \pm SEM of at least three independent experiments in which no $<100$ vacuoles were scored in each experiment $\left({ }^{*} P \leq 0.05\right)$. (I) HeLa cells were transfected with pEGFP or co-transfected with pEGFP and Beclin 1-KD plasmid using a double-hit protocol (see Experimental Procedures for details) After $24 \mathrm{~h}$, cells were infected with $\mathrm{C}$. burnetii and cultured for an additional $48 \mathrm{~h}$ period to allow the development of the large Coxiella vacuole. The cells were then lysed by sonication and the supernatant was diluted (1:100) and used to infect Vero cells. After $72 \mathrm{~h}$ of incubation (chase), cells were fixed and examined by fluorescence microscopy. Data represent the mean + SEM of at least three independent experiments where at least 1,000 cells were scored in each experiment $\left({ }^{\star *} P \leq 0.01\right)$. (J). (Top) Western blot of the assay described in (G) and (bottom) quantification of intensity of Beclin1 bands relative to actin.

supports the participation of autophagy in keeping the integrity of the Coxiella vacuole limiting membrane.

We next determined the importance of a functional autophagy pathway in Coxiella growth. To assess the role of autophagy in bacteria replication, a focus-forming unit (FFU) assay was performed as follows: MEF wt and Atg5-/- cells were infected with C. burnetii and after $24 \mathrm{~h}$ of infection, cells were scraped and lysed by sonication. Sonicates were diluted in infection medium (i.e., DMEM) and a monolayer of Vero cells were infected with a dilution of the obtained supernatant (1:100). After $72 \mathrm{~h}$ of incubation (chase), cells were fixed and examined by fluorescence microscopy (please see Experimental Procedures for more details). The FFU assay showed a marked decrease in the replicative capacity of C. burnetii in MEF Atg5-/- in comparison with wt cells, indicating an active role of autophagy in Coxiella growth (Figure 6F).

We have previously demonstrated that the autophagic protein Beclin 1 was recruited to the Coxiella replicative vacuole and that overexpression of this protein favors the development of the CRV (Vázquez and Colombo, 2010). In order to confirm the significance of the membrane damaged caused by Coxiella and the role of autophagy in this process based in the participation of Beclin1, we performed the silencing of this protein using a specific siRNA plasmid (Vázquez and Colombo, 2010). Control and Beclin 1-KD cells were infected with C. burnetii and following $48 \mathrm{~h}$ of infection, the cells were fixed, subjected to immunofluorescence against $\mathrm{Gal} 3$ and analyzed by confocal microscopy. As expected, in control cells, endogenous Gal3 was recruited to a population (20\%) of the CRVs. However, in Beclin $1-\mathrm{KD}$ cells, a much higher percentage of vacuoles decorated by endogenous Gal3 were observed (Figure 6G).

In order to determine the acidity of the vacuoles, control, and Beclin 1-KD infected cells were incubated with pHrodo green dextran and visualized by fluorescence live cell imaging. As depicted in Figure 6H, in Beclin1 KD cells, there is a decrease in the number of vacuoles labeled with the fluorogenic compound. To verify whether Beclin 1 was also involved in the replicative capacity of Coxiella, HeLa cells were transfected with a pEGFP plasmid as a control or co-transfected with a plasmid encoding a small interference RNA against Beclin 1 (pSuper Beclin 1-KD), in order to knockdown the endogenous Beclin in transfected cells (Figure 6J). After $24 \mathrm{~h}$ cells were infected with C. burnetii for additional $24 \mathrm{~h}$. The replication capacity of the bacteria was determined by the FFU assay in cells subjected to Beclin 1 knockdown. As shown in Figure 6I, left panel, a significant decrease in bacteria replication was observed when Beclin 1 was silenced which indicates that this autophagic protein is involved in the generation of an appropriate environment for bacteria growth. Taken together, these results suggest that autophagy has an important role in keeping the integrity of the membrane vacuole favoring also bacteria replication.

\section{DISCUSSION}

Galectins are molecules that bind $\beta$-galactosides at the cell surface and are also present in the cytosol, which normally lacks complex carbohydrates. Therefore, galectins may be considered as danger signal (Dupont and Lafont, 2009) and/or pattern-recognition receptors that identify pierced or broken membranes. In the present work, we report the association of galectins to the CRV, at different times of infection. All together results presented in this study support that the CRV membrane is damaged and becomes permeable to molecules like galectins that recognize glycans present in the luminal side of the vacuolar membrane. Interestingly, different members of the galectin family were recruited in a differential extent according to the infection times. We have observed that Gal3, Gal9 and Gal8 are present at the vacuole membrane in a high percentage of CRVs from early times of infection. In contrast, Gall is mainly recruited at later times of infection. This suggests that there are differences in the composition of the carbohydrates present at the inner face of the C. burnetii vacuole which are specifically recognized by each galectin. The presence of Gall at later infection times is likely due to the more mature (i.e., lysosomal) characteristics of the CRV at $48 \mathrm{~h}$. Our results are consistent with a previous publication by Randow and collaborators (Thurston et al., 2012), showing that damage of endosomes by Salmonella induces the formation of Gal3, Gal8 and Gal9 puncta, without presence of Gall. In contrast, specific damage of lysosomes results in the recruitment of Gall. Interestingly, our results indicate that Gal10 is not present at the Coxiella vacuole membrane during the whole infection period analyzed. It is known that Gal10 recognizes mannose ligands but does not recognize other type of carbohydrates such as $\mathrm{N}$-acetyl-lactosamine molecules. Thus, the lack of Gal10 recruitment at the CRV is likely due to the absence of mannose residues at the Coxiella vacuole.

As mentioned above galectins are recruited to damaged membranes by their interactions with $\mathrm{N}$-glycans that are exposed to the cytoplasm when the membrane of a vesicle is pierced. However, other intracellular functions have also been described for Gal3. It has been shown that Gal3 associates with Bcl-2 and is thought to participate in the inhibition of cellular death (Yang et al., 1996). Thus, although our present results point to Gal3 as a sensor of damage at the Coxiella limiting membrane, 
we cannot discard the possibility that Gal3 is exerting other functions at the Coxiella vacuole. Indeed, we have previously demonstrated that Bcl-2 is also recruited to the CRV and exerts a critical role in avoiding the death of the infected cells (Vázquez and Colombo, 2010). Future analyses would be necessary to actually determine other possible functions of Gal3 in Coxiella infection.

In this work, we focused on the analysis of two galectins, Gal3 and Gal8. These galectins are the best studied related to pathogens and damage of vacuole membranes, as shown in the case of Shigella (Dupont et al., 2009), Salmonella (Thurston et al., 2012), Listeria and a virus like Adenovirus (Maier et al., 2012). A pioneer work, identified the cytosolic protein Gal3 as a marker of membrane damage of phagosomes (Dupont et al., 2009; Paz et al., 2010) since the protein was recruited to membrane remnants of Shigella-containing compartments. In the case of Coxiella, we have observed that Gal3 is associated to the vacuole at the different times of infection analyzed $(6,24,48 \mathrm{~h})$. We have also demonstrated that the recruitment of galectins depends on the presence of mature glycans from the host cell and that the recognition of these glycans by galectins depends on intact CRDs. It is important to mention that our results also indicate that recruited galectins comes from the cytosol and not from the plasma membrane during the process of Coxiella internalization.

There is evidence that autophagy may function as a selective pathway that degrades protein aggregates and organelles. It has been shown that ubiquitination in conjunction with adapter proteins such as p62/SQSTM1, which allows the interaction between LC3 and ubiquitin, participates in the targeting to autophagy. We have shown the presence of host p62 and ubiquitin on CRV membranes suggesting that C. burnetii interacts with the host ubiquitination-autophagy interface during intracellular growth. Our results about the association of p62 to the CRV membrane are in agreement with recent findings showing the recruitment of p62 to the Coxiella parasitophorous vacuole (Winchell et al., 2014). Interestingly, this recruitment and the LC3 association, were dependent on a functional type IV secretion system (Winchell et al., 2014), which is also consistent with our observations that the recruitment of LC3 (Romano et al., 2007) and galectins (this report), is abolished by chloramphenicol treatment which hampers bacterial protein synthesis. We have also demonstrated here that a type IV secretion mutant was unable to recruit galectins to the Coxiella vacuole.

Our present results are also consistent with earlier report showing that the membrane remnants released after the rupture of Shigella-containing vacuolar membranes undergo protein polyubiquitination and subsequently are targeted to autophagy (Dupont et al., 2010). The role of p62 and/or NDP52 in selective autophagy of Salmonella enterica serovar Typhimurium (S. typhimurium) has recently been characterized (Zheng et al., 2009; Mostowy et al., 2011). It has been proposed that p62 and NDP52 act independently to drive efficient bacterial autophagy of S. typhimurium within Salmonella-containing vacuoles (Verlhac et al., 2015). In this work, we have shown that a population of Coxiella vacuoles is marked by NDP52, with a pattern very similar to ubiquitin. It remains to be determined if similar to
Salmonella both adaptors participate independently or not to mediate the interaction with the autophagy pathway.

It is evident that once the CRV membrane is damaged or pierced, the vacuole would lose the protons and the acidic characteristics. To assess vacuole acidification, we have utilized LysoSensor and pHrodo, a novel fluorogenic dye that dramatically increases in fluorescence as the $\mathrm{pH}$ becomes more acidic. Our experiments using pHrodo directly demonstrate that different populations of CRVs exist. Indeed, some of them displayed high fluorescence intensity but some were not stained. These differences were not dependent on vacuole diameter and were not dependent on the presence or the absence of autophagy markers (i.e., LC3, not shown). Thus, our results suggest that some vacuoles have lost their protons and, as a consequence, they are not labeled by pHrodo. The permeability of the Coxiella membrane vacuole was demonstrated by the microinjection of small molecules such as $3 \mathrm{kDa}$ dextran, in the cytoplasm of cells infected with C. burnetii. Our results indicate that a fraction of the Coxiella vacuoles were permeable to the cytoplasmic microinjected dextran as demonstrated by the accumulation of the fluorescent compound inside the vacuole. It is interesting to mention that in a publication by David Russell and collaborators, the authors found that dextran particles previously loaded into cells, were transferred from the cytoplasm to the Leishmania mexicana parasitophorous vacuole likely via an autophagy-dependent mechanism. However, this process required prolonged incubation times (i.e., $10 \mathrm{~h}$ ) since at $4 \mathrm{~h}$ post-loading the dextrans remained mostly cytosolic (Schaible et al., 1999). Also, in a publication of Heinzen and Hackstadt (1997), Vero cells with large PVs harboring C. burnetii were microinjected with probes as small as $623 \mathrm{Da}$ showing the CRV is impermeable to them. In contrast, in our system, the transfer of fluorescent dextran to the Coxiella vacuole was almost immediately after the microinjection into the cytoplasm which is more compatible with a damage of the Coxiella membrane. In addition, we have demonstrated by using a modified FRET assay, based in the detection of $\beta$-lactamase activity when the cytoplasm gets in contact with internalized beads coated with this enzyme, that the integrity of the CRV is altered in Coxiella infected cells.

It has been shown that lysosomes are also marked with Gal3 when they are damaged and, as a consequence, can be targeted to autophagy (Maejima et al., 2013). The induction of autophagy by damaged lysosomal compartments occurs after the loss of acidic content and release of lysosomal cathepsin D. This last evidence points to a role of autophagy in the sequestration of damaged lysosomes in order to allow the isolation of harmful material that may induce inflammatory responses. Indeed, it has been observed membranes around the lysosomes, suggesting that canonical autophagy eliminates damaged lysosomes. Once the rupture of the lysosome happens, the autophagosomes would selectively hijack damaged lysosomes. Ubiquitin is likely involved in the recognition of these damaged lysosomes. It is also feasible that autophagosomes fuse with lysosomes and restore the damaged compartments by repairing the holes in the membrane, resulting in the recovery of the acidic $\mathrm{pH}$. This mechanism was also demonstrated in a Salmonella infection system. In a recent 
publication of Kreibich et al, it has been shown that autophagy participates in the repair of SCV membrane damage (Kreibich et al., 2015). This autophagy-dependent SCV sealing requires recruitment of factors such as optineurin and galectin 3 and the autophagy initiator factors ULK1, Beclin1, and ATG9, suggesting that LC3 is recruited to SCV by a canonical pathway (Kreibich et al., 2015).

Thus, we propose that a similar mechanism may take place in Coxiella infected cells, allowing the vacuole to restore its acidic properties and degradative capacity. Indeed, we propose herein that $C$. burnetii's vacuole might restore its acidic environment by resealing the membrane. We have presented evidence that in MEF cells KO for Atg5, a critical protein involved in autophagy, a larger number of Gal3-labeled CRVs are detected, suggesting an increase in the number of damaged Coxiella vacuoles. In agreement with this, we have also found, based on the use of the pHrodo marker, a decrease in the number of Coxiella-containing acidic vacuoles. Therefore, our present results support a role for autophagy in membrane repair contributing to the integrity of the Coxiella replicative vacuoles.

In summary, in the present report we have uncovered an unexpected outcome of the Coxiella infection which is the damage of the Coxiella-containing vacuole causing that several CRVs do not maintain its acidic $\mathrm{pH}$. We propose that autophagy may contribute to the resealing of the damaged membrane likely via fusion with autophagosomes and autolysosomes. Further studies are necessary to elucidate the molecular machinery involved in the damage and in these fusion events leading to the repair of the injured CRV membrane. Our study paves the way to investigate whether this phenomenon is also involved in other infectious process and beyond the infection field how general is the role of autophagy in membrane repair mechanisms.

\section{EXPERIMENTAL PROCEDURES}

\section{Materials}

Minimum essential Medium Alpha Medium (alfa-MEM) and Dulbecco's Modified Eagle Medium (D-MEM) were obtained from Gibco Laboratories (Invitrogen, Argentina); fetal bovine serum (FBS) was obtained from GIBCO BRL/Life Technologies (Buenos Aires, Argentina). Rabbit polyclonal anti-Coxiella antibody and mCherry-Coxiella burnetii were generously provided by Dr. Robert Heinzen (Rocky Mountain Laboratories, NIAID, NIH, Hamilton, MT, USA). Plasmids encoding YFPGal4, YFP-Gal9, YFP-Gal3, YFP-Gal10, YFP-Gal1, YFP-Gal8, and RFP-NDP52 were kindly provided by Dr. Felix Randow (MRC Laboratory of Molecular Biology, Hills Road, Cambridge, CB2 0QH, U.K.). Rabbit and mouse polyclonal antibodies antiGal3, -Gal1, -Gal8 and a plasmid encoding Gal3 were generously provided by Dr. Gabriel Rabinovich (Instituto de Biología y Medicina Experimental, INYME-CONICET, Buenos Aires, Argentina). The Beclin $1 \mathrm{KD}$ plasmid was a generous gift of Dr. William Maltese (Medical University of Ohio, Toledo, Ohio, USA). Tandem fluorescent-tagged Galectin-3, tfGal3 was kindly provided by Dr. Tamotsu Yoshimori (Laboratory of Intracellular Membrane Dynamics, Graduate School of Frontier Biosciences, Osaka University, Osaka, Japan). mCherry-Gal8 was kindly provided by Dr. Mauricio Terebiznik (Department of Biological Sciences, University of Toronto, Toronto, Canada). Coxiella-GFP (Tn1832) and DotA mutant GFP (Tn292) were kindly provided by Dr. Matteo Bonazzi (Cell Biology of Bacterial Infections, UMR 5236 CPBS, Montpellier, France). CHO-Lec3.2.8.1 cells were kindly provided by Dr Pamela Stanley (Albert Einstein College of Medicine, New York, USA). Ubiquitin antibody was from Abcam.

\section{Fluorophore}

Texas Red-tagged dextran $(3 \mathrm{kDa})$, pHrodo ${ }^{\mathrm{TM}}$ Succinimidyl Ester, pHrodo ${ }^{\mathrm{TM}}$ Green dextran, 10,000 MW, LysoTracker, LysoSensor green DND-189 and DQ-BSA were purchased from Molecular Probes.

\section{Cell Culture}

Vero (ATCC, CCL-81), MEF (ATCC, SCRC-1040), and Chinese hamster ovary cells (CHO; ATCC, CCL-61) were grown on coverslips in D-MEM or $\alpha$-MEM supplemented with $15 \%$ FBS, at $37^{\circ} \mathrm{C}$ in an atmosphere of $95 \%$ air and $5 \% \mathrm{CO} 2$, in 24-well-plates to $80 \%$ confluence.

\section{Cell Transfection}

MEF and CHO cells were transfected with the plasmids $(1 \mu \mathrm{g} / \mu \mathrm{l})$ using LipofectAMINE 2000 reagent (Invitrogen, Argentina) as previously described in Campoy et al. (2013). Transfected cells were incubated for $24 \mathrm{~h}$ in DMEM and were infected with C. burnetii as described above.

\section{Propagation of Coxiella burnetii Phase II Cells}

Obtaining C. burnetii was performed as previously described in Romano et al. (2007). Briefly, C. burnetii clone 4, phase II, strain Nine Mile bacteria were provided by Ted Hackstadt (Rocky Mountain Laboratories, NIAID, NIH, Hamilton, MT) and handled in a biosafety level II facility. For initial infection, $80 \%$ confluent Vero cells were infected with C. burnetii for 2 weeks at $37^{\circ} \mathrm{C}$ under $5 \% \mathrm{CO} 2$. C. burnetii were obtained from infected cells by lysis of the host cells followed a differential centrifugation of the supernatants. Purified C. burnetii in the resulting pellet were resuspended in PBS and frozen at $-70^{\circ} \mathrm{C}$.

\section{Infection of Cells with C. burnetii}

Cells were plated on coverslips distributed in 6 or 24 well-plates and $0.5-1 \mathrm{ml}$ of a dilution of $C$. burnetii suspension was added per well. Afterwards the cells were incubated at $37^{\circ} \mathrm{C}$ in an atmosphere of $95 \%$ air and $5 \% \mathrm{CO} 2$ for the indicated time periods as described (Campoy et al., 2013).

\section{Ectopic Expression and Confocal Microscopy}

GFP-LC3, YFP-Gal8, YFP-Gal3, YFP-Gal9, YFP-Gal10, YFPGal4, YFP-Gal, YFP-Gal8 (R275H), YFP-Gal8 (R69H), and GFP-Gal3 (R186S) or RFP-NDP52 transfected CHO cells were analyzed by confocal microscopy using an Olympus FluoViewTM FV1000 confocal microscope (Olympus, Argentina), with the FV10-ASW (version 01.07.00.16) software. 
Images were processed using Adobe CS3 (Adobe Systems). Confocal images $(0.39-\mu \mathrm{m}$ sections) were collected.

\section{Super-Resolution Microscopy}

High-resolution images were acquired on a ElyraPS1 microscope system (Zeiss, Carl Zeiss-Strasse 2273447 Oberkochen, Germany) using a 100 oil-immersion lens (NA 1.46). This SIM system can achieve a resolution of $100 \mathrm{~nm}$ along the $\mathrm{x}-\mathrm{y}$ axis and $300 \mathrm{~nm}$ along the $\mathrm{z}$-axis. Laser lines at 488 and $561 \mathrm{~nm}$ were used for excitation. SIM images (15 images with five different phases for 3 different angular orientations of illumination for each SIM image) were acquired with an EMCCD camera (Andor Technology Ltd., Millennium WaySpringvale Business Park, Belfast BT12 7AL, UK; 1,002 1,004 pixels) and processed with Zen (Zeiss) software.

\section{Video-Microscopy}

Some in vivo experiments were recorded using an inverted AxioObserver $\mathrm{Z} 1$ microscope fitted with a Zeiss Axiocam ${ }^{\circledR} \mathrm{MRm}$ camera (Carl Zeiss, Oberkochen, Germany) and operated by Axiovision ${ }^{\circledR}$ software. Images were acquired with a Plan-Apo $100 \times / 1.46$ oil immersion objective (Carl Zeiss, Oberkochen, Germany). For video microscopy, the fluorophore excitation system was composed of a Colibri system (Zeiss, Carl Zeiss, Oberkochen, Germany) with 365, 470, 555, and $590 \mathrm{~nm}$ LEDs. For $\mathrm{pH}$ measurements, images were adquired in a LEICA fluorescence microscope spinning disk (AF6000-X; Campus CNRS CCHB, Bio Imaging Center Lille-Campus Lille 1, Université de Lille)

\section{Indirect Immunofluorescence}

The cells grown on coverslips were fixed with $3 \%$ paraformaldehyde solution in PBS for $10 \mathrm{~min}$ at room temperature, washed with PBS followed by quenching with $50 \mathrm{mM} \mathrm{NH} 4 \mathrm{Cl}$ in PBS. Subsequently, cells were permeabilized with $1 \%$ saponin in PBS containing $1 \%$ BSA, and further incubated with the primary antibody dilution in PBS. Afterwards, the coverslips were incubated with a conjugated secondary antibody (Jackson immune Research Laboratories, EE. UU). After $3 \mathrm{x}$ washing with PBS cells were mounted with Mowiol (plus Hoechst) and examined by confocal microscopy as described previously (Campoy et al., 2013).

\section{Microinjection of Infected CHO Cells}

Infected $\mathrm{CHO}$ cells were injected as previously described (Teitelbaum et al., 1999) with $3 \mathrm{kDa}$ Texas red dextran, using a Micromanipulator 5,171 and a Transjector 5,426 plus (Eppendorf). Needles were pulled on a Sutter pipette puller, model P97. Infected $\mathrm{CHO}$ cells were microinjected with an initial pressure of $600 \mathrm{k} \mathrm{Pa}$. Successive optical sections were collected and analyzed in a spin disk microscope (Olympus). The $\mathrm{z}$ series collection was initiated once diffusion of the injected marker was determined by imaging fluorescence. Injected cells were imaged for 20 min immediately after microinjection.

\section{pHrodo Coupled to S. aureus Assay}

Preparations of Staphylococcus aureus particles conjugated to pHrodo were performed as previously described (Kobayashi et al., 2010). Heat-dead bacteria were washed twice and resuspended in PBS at an appropriate concentration (1:10 or 1:20 dilution). pHrodo-succinimidyl ester (Invitrogen, P36600) was added to the bacteria suspension and the samples were incubated in buffer bicarbonate for $45 \mathrm{~min}$ at room temperature. After conjugation, bacteria were washed and finally suspended in PBS. The resulting suspension $(\sim 10 \mu \mathrm{l})$ was mixed with $90 \mu \mathrm{l}$ of culture medium and added to cells by replacing the medium. After incubation with the mixture for $1 \mathrm{~h}$ at $37^{\circ} \mathrm{C}$, cells were washed with fresh medium to remove particles or not phagocytosed bacteria, and cells were subsequently incubated for $2 \mathrm{~h}$.

\section{Beclin 1 Knockdown}

A double-hit protocol was performed as previously described in Vázquez and Colombo (2010). HeLa cells were plated in sixwell-plates at $40 \%$ confluence and then transfected with GFP (control) or GFP and Beclin 1-KD (plasmid) After $24 \mathrm{~h}$, cells were transfected with $24 \mathrm{~h}$ later, cells were transfected again with the GFP (control) or GFP and Beclin 1-KD (plasmid) again. Twenty four hours later cells were infected with C. burnetii during $48 \mathrm{~h}$.

\section{Bacterial Viability and Replication}

A fluorescent infectious FFU assay was used to quantify the replication and viability of C. burnetii in MEF wt or Atg5-/cells or in Beclin $1 \mathrm{KD}$ HeLa cells. In brief, the infected cells were lysed and samples were serially diluted. Vero cells were infected with these lysates in a 24 well-plate. After $72 \mathrm{~h}$ of infection, Vero cells were fixed and processed for fluorescent microscopy. Approximately 1,000 cells were scored per coverslip to determine an average number of FFU for each sample.

\section{pH Measurements}

For standard curves, C. burnetii infected cells were incubated with $10 \mathrm{mM}$ nigericin and $10 \mathrm{mM}$ valinomycin in the $\mathrm{pH} 7.0$ calibration Buffer. The coverslips were subsequently incubated for $10 \mathrm{~min}$ in $\mathrm{pH}$ calibration buffers adjusted to $\mathrm{pH}$ of 4.0, $5.0,6.0$, or 7.0. Then, they were incubated with Oregon green dextran 10,000 MW at a concentration of $1 \mathrm{mg} / \mathrm{ml}$ in culture medium overnight. Images for ratiometric calculations were acquired in a Leica spinning disk at excitation wavelengths of $440 \mathrm{~nm}$ and $480 \mathrm{~nm}$ with a fixed emission wavelength of $520 \mathrm{~nm}$. Ratio values for the standard curves were determined by measuring the average $480 / 440$ ratio value. To determine the $\mathrm{pH}$ of $C$. burnetii's vacuoles, infected cells were incubated with Oregon green dextran in culture medium overnight. Ratio values were plotted against the standard curves to determine the $\mathrm{pH}$ of C. burnetii replicative vacuoles.

\section{Coating of Beads with Beta-Lactamase}

Amine functionalized beads were incubated for $4 \mathrm{~h}$ in $8 \%$ glutaraldehyde in PBS on a rolling wheel at RT. Activated beads were washed and resuspended in lactamase containing solutions in saturating conditions (estimated following the manufacturer's instructions). They were left overnight on a rolling wheel at $4^{\circ} \mathrm{C}$. Beads were then washed and stocked at $4^{\circ} \mathrm{C}$ in PBS. 


\section{CCF4 Assay for Coxiella Vacuole Rupture}

$\mathrm{CHO}$ cells were seeded on coverslips in 24 -well plates at $2 \times 10^{4}$ cells per well for experiments with fixed samples. CHO cells were infected with $C$. burnetii and after $48 \mathrm{~h}$, they were loaded with $0.1 \mathrm{mM}$ CCF4/AM substrate (Invitrogen) in EM buffer $(120 \mathrm{mM}$ $\mathrm{NaCl}, 7 \mathrm{mM} \mathrm{KCl}, 1.8 \mathrm{mM}, 0.8 \mathrm{mM} \mathrm{MgCl} 2,5 \mathrm{mM}$ glucose and $25 \mathrm{mM}$ Hepes, $\mathrm{pH} 7.3$ ) containing $6 \mathrm{mM}$ probenecid, an inhibitor of anions transporters, for $2 \mathrm{~h}$ at RT, washed twice with EM and transferred to fresh medium containing $6 \mathrm{mM}$ probenecid for a further $30 \mathrm{~min}$. CCF4-loaded $\mathrm{CHO}$ cells were directly incubated with beta-lactamase coated beads (see Coating of beads with lactamase) for $8 \mathrm{~h}$ at $37^{\circ} \mathrm{C}$. The adquisition was performed with a spinning-disk confocal microscope is composed of two diode laser sources emitting at wavelength of 488 and $440 \mathrm{~nm}$ (Omicron, Germany) injected in a spinning-disk confocal system (Yokogawa CSU-X1, Tokyo, Japan) adapted on a Leica (Lognes, FR) DMI6000B inverted microscope. Cells were imaged using a $63 \times$ water-immersion objective (Leica HCX Plan Apo NA $1.20 \mathrm{~W}$ Corr CS). Fluorescence emitted was then successively routed by a dichroic mirror (Semrock Di01-T405/488/568/647 and 405/440/514 respectivlely), spectrally filtered (Semrock, FF01-520/35 nm) and detected with a Quatem 512SC EMCCD coupled to an optical zoom $(\times 2)$. Every $512 \times 512$ image was acquired using $300 \mathrm{~ms}$ exposure time. The acquisition was performed by separated spectra to avoid potential overlap between emissions. Results were analyzed using ImageJ software (live cell imaging).

\section{AUTHOR CONTRIBUTIONS}

$\mathrm{MM}$ and $\mathrm{AB}$ Methodology and Investigation. $\mathrm{MM}, \mathrm{FL}$, and $\mathrm{MC}$ Writing-Review and Editing. FL and MC Funding Acquisition, Resources, Supervision.

\section{REFERENCES}

Akporiaye, E. T., Rowatt, J. D., Aragon, A. A., and Baca, O. G. (1983). Lysosomal response of a murine macrophage-like cell line persistently infected with Coxiella burnetii. Infect Immun. 40, 1155-1162.

Beare, P. A., Larson, C. L., Gilk, S. D., and Heinzen, R. A. (2012). Two systems for targeted gene deletion in Coxiella burnetii. Appl. Environ. Microbiol. 78, 4580-4589. doi: 10.1128/AEM.00881-12

Beare, P. A., Unsworth, N., Andoh, M., Voth, D. E., Omsland, A., Gilk, S. D., et al. (2009). Comparative genomics reveal extensive transposon-mediated genomic plasticity and diversity among potential effector proteins within the genus Coxiella. Infect. Immun. 77, 642-656. doi: 10.1128/IAI.01141-08

Berón, W., Gutierrez, M. G., Rabinovitch, M., and Colombo, M. I. (2002). Coxiella burnetii localizes in a Rab7-labeled compartment with autophagic characteristics. Infect Immun. 70, 5816-5821. doi: 10.1128/IAI.70.10.5816-5821.2002

Birmingham, C. L., and Brumell, J. H. (2006). Autophagy recognizes intracellular Salmonella enterica serovar Typhimurium in damaged vacuoles. Autophagy 2, 156-158. doi: 10.4161/auto.2825

Bjørkøy, G., Lamark, T., Brech, A., Outzen, H., Perander, M., Overvatn, A., et al. (2005). p62/SQSTM1 forms protein aggregates degraded by autophagy and has a protective effect on huntingtin-induced cell death. J. Cell Biol. 171, 603-614. doi: $10.1083 /$ jcb.200507002

Campoy, E. M., Mansilla, M. E., and Colombo, M. I. (2013). Endocytic SNAREs are involved in optimal Coxiella burnetii vacuole development. Cell Microbiol. 15, 922-941. doi: $10.1111 / \mathrm{cmi} .12087$

\section{ACKNOWLEDGMENTS}

We are grateful to Dr. Robert Heinzen (Rocky Mountain Laboratories, NIAID/NIH, Hamilton, Montana, USA) for generously providing mCherry Coxiella burnetii. We also thank Matteo Bonazzi for providing GFP-C. burnetii and the corresponding DotA mutant. We thank Dr. Felix Randow (MRC Laboratory of Molecular Biology, Hills Road, Cambridge, CB2 0QH, U.K.) for the plasmids encoding YFP-Gal4, YFP-Gal9, YFPGal3, YFP-Gal10, YFP-Gal1, YFP-Gal8, and RFP-NDP52. We are also grateful to Dr. Gabriel Rabinovich (Instituto de Biología y Medicina Experimental INYME-CONICET, Buenos Aires) and to Giannina Pasquini (UBA) for the generous gift of rabbit and mouse polyclonal antibodies anti-Gal3,-Gal1, -Gal8, and plasmid encoding Gal3. We are indebted to Dr. Alfredo Cáceres (Instituto Martín Ferreyra, Córdoba, Argentina) for helping us to perform the microinjection experiments in his laboratory. We would like to thank Dr. Luis Mayorga and Dr. Walter Berón for critically reading of this manuscript. We also thank Alejandra Medero and Rodrigo Militello for excellent technical assistance with tissue culture and Jorge Ibañez for valuable technical assistance with confocal and electron microscopy handling. We thank Dr. Jost Enninga for sharing the $\beta$-lactamase beads and advices on the FRET assay. We thank the BioImaging Center Lille Facility for access to systems and advices and $\mathrm{N}$ Barois for EM. This study was supported by funds from CoopIntEER-254429-CNRS to FL and by the grants SeCTyP 06/J432 PICT2011-0455 and PICT2013-0305 to MC. MM is a CONICET fellow.

\section{SUPPLEMENTARY MATERIAL}

The Supplementary Material for this article can be found online at: http://journal.frontiersin.org/article/10.3389/fcimb. 2017.00112/full\#supplementary-material

Campoy, E. M., Zoppino, F. C., and Colombo, M. I. (2011). The early secretory pathway contributes to the growth of the Coxiella-replicative niche. Infect. Immun. 79, 402-413. doi: 10.1128/IAI.00688-10

Carey, K. L., Newton, H. J., Lührmann, A., and Roy, C. R. (2011). The Coxiella burnetii Dot/Icm system delivers a unique repertoire of type IV effectors into host cells and is required for intracellular replication. PLoS Pathog. 7:e1002056. doi: 10.1371/journal.ppat.1002056

Dupont, N., Lacas-Gervais, S., Bertout, J., Paz, I., Freche, B., Van Nhieu, G. T., et al. (2009). Shigella phagocytic vacuolar membrane remnants participate in the cellular response to pathogen invasion and are regulated by autophagy. Cell Host Microbe 6, 137-149. doi: 10.1016/j.chom.2009. 07.005

Dupont, N., and Lafont, F. (2009). How autophagy regulates the host cell signaling associated with the postpartum bacteria cocoon experienced as a danger signal. Autophagy 5, 1222-1223. doi: 10.4161/auto.5.8.10218

Dupont, N., Temime-Smaali, N., and Lafont, F. (2010). How ubiquitination and autophagy participate in the regulation of the cell response to bacterial infection. Biol. Cell. 102, 621-634. doi: 10.1042/BC20100101

Grieshaber, S., Swanson, J. A., and Hackstadt, T. (2002). Determination of the physical environment within the Chlamydia trachomatis inclusion using ion-selective ratiometric probes. Cell. Microbiol. 4, 273-283. doi: 10.1046/j.1462-5822.2002.00191.x

Gutierrez, M. G., Vázquez, C. L., Munafó, D. B., Zoppino, F. C., Berón, W., Rabinovitch, M., et al. (2005). Autophagy induction favours the generation and maturation of the Coxiella-replicative vacuoles. Cell Microbiol. 7, 981-993. doi: 10.1111/j.1462-5822.2005.00527.x 
Heinzen, R. A., and Hackstadt, T. (1997). The Chlamydia trachomatis parasitophorous vacuolar membrane is not passively permeable to lowmolecular-weight compounds. Infect. Immun. 65, 1088-1094.

Heinzen, R. A., Hackstadt, T., and Samuel, J. E. (1999). Developmental biology of Coxiella burnettii. Trends Microbiol. 7, 149-154. doi: 10.1016/S0966-842X(99)01475-4

Heinzen, R. A., Scidmore, M. A., Rockey, D. D., and Hackstadt, T. (1996). Differential interaction with endocytic and exocytic pathways distinguish parasitophorous vacuoles of Coxiella burnetii and Chlamydia trachomatis. Infect Immun. 64, 796-809.

Keller, C., Mellouk, N., Danckaert, A., Simeone, R., Brosch, R., Enninga, J., et al. (2013). Single cell measurements of vacuolar rupture caused by intracellular pathogens. J. Vis. Exp. 76:e50116. doi: 10.3791/50116

Kobayashi, S., Kojidani, T., Osakada, H., Yamamoto, A., Yoshimori, T., Hiraoka, Y., et al. (2010). Artificial induction of autophagy around polystyrene beads in nonphagocytic cells. Autophagy 6, 36-45. doi: 10.4161/auto.6.1.10324

Kohler, L. J., Reed, S. R., Sarraf, S. A., Arteaga, D. D., Newton, H. J., and Roy, C. R. (2016). Effector protein Cig2 decreases host tolerance of infection by directing constitutive fusion of autophagosomes with the Coxiella-containing vacuole. mBio 7, 1-14. doi: 10.1128/mBio.01127-1

Komatsu, M., and Ichimura, Y. (2010). Physiological significance of selective degradation of p62 by autophagy. FEBS Lett. 584, 1374-1378. doi: 10.1016/j.febslet.2010.02.017

Kreibich, S., Emmenlauer, M., Fredlund, J., Rämö, P., Münz, C., Dehio, C., et al. (2015). Autophagy proteins promote repair of endosomal membranes damaged by the salmonella type three secretion system 1. Cell Host. Microbe. 18, 527-537. doi: 10.1016/j.chom.2015.10.015

Maejima, I., Takahashi, A., Omori, H., Kimura, T., Takabatake, Y., Saitoh, T., et al. (2013). Autophagy sequesters damaged lysosomes to control lysosomal biogenesis and kidney injury. EMBO J. 32, 2336-2347. doi: 10.1038/emboj.2013.171

Maier, O., Marvin, S. A., Wodrich, H., Campbell, E. M., and Wiethoff, C. M. (2012). Spatiotemporal dynamics of adenovirus membrane rupture and endosomal escape. J. Virol. 86, 10821-10828. doi: 10.1128/JVI.01428-12

Maurin, M., Benoliel, A. M., Bongrand, P., and Raoult, D. (1992). Phagolysosomal alkalinization and the bactericidal effect of antibiotics: the Coxiella burnetii paradigm. Infect. Immun. 166, 1097-1102. doi: 10.1093/infdis/166. 5.1097

McDonough, J. A., Newton, H. J., Klum, S., Swiss, R., Agaisse, H., and Roy, C. R. (2013). Host pathways important for Coxiella burnetii infection revealed by genome-wide RNA interference screening. mBio 4:e00606-12. doi: $10.1128 / \mathrm{mBio} .00606-12$

Mostowy, S., Sancho-Shimizu, V., Hamon, M. A., Simeone, R., Brosch, R., Johansen, T., et al. (2011). p62 and NDP52 proteins target intracytosolic Shigella and Listeria to different autophagy pathways. J. Biol. Chem. 286, 26987-26995. doi: 10.1074/jbc.M111.223610

Munafó, D. B., and Colombo, M. I. (2002). Induction of autophagy causes dramatic changes in the subcellular distribution of GFP-Rab24. Traffic 3, 472-482. doi: 10.1034/j.1600-0854.2002.30704.x

Newton, H. J., Kohler, L. J., McDonough, J. A, Temoche-Diaz, M., Crabill, E., Hartland, E. L., et al. (2014). A screen of Coxiella burnetii mutants reveals important roles for Dot/Icm effectors and host autophagy in vacuole biogenesis. PLoS Pathog. 10:e1004286. doi: 10.1371/journal.ppat.1004286

Newton, H. J., and Craig, R. R. (2011). The Coxiella burnetii Dot/Icm system creates a comfortable home through lysosomal renovation. mBio 2:e0226-11. doi: 10.1128/mBio.00226-11

Paz, I., Sachse, M., Dupont, N., Mounier, J., Cederfur, C., Enninga, J., et al. (2010). Galectin-3, a marker for vacuole lysis by invasive pathogens. Cell Microbiol. 12, 530-544. doi: 10.1111/j.1462-5822.2009.01415.x

Perrin, A. J., Jiang, X., Birmingham, C. L., So, N. S., and Brumell, J. H. (2004). Recognition of bacteria in the cytosol of mammalian cells by the ubiquitin system. Curr Biol. 14, 806-811. doi: 10.1016/j.cub.2004.04.033

Rabinovich, G. A., and Toscano, M. A. (2009). Turning "sweet" on immunity: galectin-glycan interactions in immune tolerance and inflammation. Nat. Rev. Immunol. 9, 338-352. doi: 10.1038/nri2536

Ray, K., Bobard, A., Danckaert, A., Paz-Haftel, I., Clair, C., Ehsani, S., et al. (2010). Tracking the dynamic interplay between bacterial and host factors during pathogen-induced vacuole rupture. Cell. Microbiol. 12, 545-556. doi: 10.1111/j.1462-5822.2010.01428.x

Romano, P. S., Gutierrez, M. G., Berón, W., Rabinovitch, M., and Colombo, M. I. (2007). The autophagic pathway is actively modulated by phase II Coxiella burnetii to efficiently replicate in the host cell. Cell Microbiol. 9, 891-909. doi: 10.1111/j.1462-5822.2006.00838.x

Sauer, J. D., Shannon, J. G., Howe, D., Hayes, S. F., Swanson, M. S., and Heinzen, R. A. (2005). Specificity of Legionella pneumophila and Coxiella burnetii vacuoles and versatility of Legionella pneumophila revealed by coinfection. Infect. Immun. 73, 4494-4504. doi: 10.1128/IAI.73.8.4494-4504.2005

Schaible, U. E., Schlesinger, P. H., Steinberg, T. H., Mangel, W. F., Kobayashi, T., and Russell, D. G. (1999). Parasitophorous vacuoles of Leishmania mexicana acquire macromolecules from the host cell cytosol via two independent routes. J. Cell Sci. 112, 681-693.

Shibutani, S. T., and Yoshimori, T. (2014). A current perspective of autophagosome biogenesis. Cell Res. 24, 58-68. doi: 10.1038/cr.2013.159

Simeone, R., Bobard, A., Lippmann, J., Bitter, W., Majlessi, L., Brosch, R., et al. (2012). Phagosomal rupture by Mycobacterium tuberculosis results in toxicity and host cell death. PLoS Pathog. 8:e1002507. doi: 10.1371/journal.ppat.1002507

Tanida, I. (2011). Autophagosome formation and molecular mechanism of autophagy. Antioxid. Redox Signal. 14, 2201-2214. doi: 10.1089/ars.2010.3482

Teitelbaum, R., Cammer, M., Maitland, M. L., Freitag, N. E., Condeelis, J., and Bloom, B. R. (1999). Mycobacterial infection of macrophages results in membrane-permeable phagosomes. Proc. Natl. Acad. Sci. U.S.A. 96, 15190-15195. doi: 10.1073/pnas.96.26.15190

Thurston, T. L. M., Ryzhakov, G., Bloor, S., von Muhlinen, N., and Randow, F. (2009). The TBK1 adaptor and autophagy receptor NDP52 restricts the proliferation of ubiquitin-coated bacteria. Nat Immunol. 10, 1215-1221. doi: $10.1038 /$ ni. 1800

Thurston, T. L. M., Wandel, M. P., von Muhlinen, N., Foeglein, A., and Randow, F. (2012). Galectin 8 targets damaged vesicles for autophagy to defend cells against bacterial invasion. Nature 482, 414-418. doi: 10.1038/nature10744

Vasta, G. R. (2013). Roles of galectins in infection. Nat Rev Microbiol. 7, 424-438. doi: $10.1038 /$ nrmicro2146

Vázquez, C. L., and Colombo, M. I. (2010). Coxiella burnetii modulates Beclin 1 and $\mathrm{Bcl}-2$, preventing host cell apoptosis to generate a persistent bacterial infection. Cell Death Differ. 17, 421-438. doi: 10.1038/cdd.2009.129

Verlhac, P., Grégoire, I. P., Azocar, O., Petkova, D. S., Baguet, J., Viret, C., et al. (2015). Autophagy receptor NDP52 regulates pathogencontaining autophagosome maturation. Cell Host Microbe. 17, 515-525. doi: 10.1016/j.chom.2015.02.008

Voth, D. E., and Heinzen, R. A. (2007). Lounging in a lysosome: the intracellular lifestyle of Coxiella burnetii. Cell. Microbiol. 9, 829-840. doi: 10.1111/j.1462-5822.2007.00901.x

Winchell, C. G., Graham, J. G., Kurten, R. C., and Voth, D. E. (2014). Coxiella burnetii type IV secretion-dependent recruitment of macrophage autophagosomes. Infect. Immun. 82, 2229-2238. doi: 10.1128/IAI.01236-13

Yang, R. Y., Hsu, D. K., and Liu, F. T. (1996). Expression of galectin-3 modulates T-cell growth and apoptosis. Proc. Natl. Acad. Sci. U.S.A. 93, 6737-6742. doi: 10.1073/pnas.93.13.6737

Zheng, Y. T., Shahnazari, S., Brech, A., Lamark, T., Johansen, T., and Brumell, J. H. (2009). The adaptor protein p62/SQSTM1 targets invading bacteria to the autophagy pathway. J. Immunol. 183, 5909-5916. doi: 10.4049/jimmunol.0900441

Conflict of Interest Statement: The authors declare that the research was conducted in the absence of any commercial or financial relationships that could be construed as a potential conflict of interest.

Copyright (c) 2017 Mansilla Pareja, Bongiovanni, Lafont and Colombo. This is an open-access article distributed under the terms of the Creative Commons Attribution License (CC BY). The use, distribution or reproduction in other forums is permitted, provided the original author(s) or licensor are credited and that the original publication in this journal is cited, in accordance with accepted academic practice. No use, distribution or reproduction is permitted which does not comply with these terms. 